\title{
Labyrinthe
}

25 | 2006 (3)

La Bande dessinée : ce qu'elle dit, ce qu'elle montre

\section{Du voyou au critique : parler de la Bande dessinée} (par Dusquier et Pabreuil)

\section{Laurent Dubreuil et Renaud Pasquier}

\section{(2) OpenEdition}

Journals

Édition électronique

URL : http://journals.openedition.org/labyrinthe/4071

DOI : $10.4000 /$ labyrinthe.4071

ISSN : 1950-6031

Éditeur

Hermann

\section{Édition imprimée}

Date de publication : 1 décembre 2006

Pagination : 11-59

ISBN : 2-9526131-2-5

\section{Référence électronique}

Laurent Dubreuil et Renaud Pasquier, « Du voyou au critique : parler de la Bande dessinée », Labyrinthe [En ligne], 25 | 2006 (3), mis en ligne le 28 mars 2010, consulté le 30 avril 2019. URL : http:// journals.openedition.org/labyrinthe/4071 ; DOI : 10.4000/labyrinthe.4071

Ce document a été généré automatiquement le 30 avril 2019.

Propriété intellectuelle 


\title{
Du voyou au critique : parler de la Bande dessinée
}

\author{
(par Dusquier et Pabreuil)
}

Laurent Dubreuil et Renaud Pasquier

Il faudrait faire un dessin.

2 Une caricature de presse à l'ancienne (d'un journal américain: nous sommes, pour l'instant, aux États-Unis), visages stylisés, expressions outrées, gros traits foncés. Elle représenterait une salle, vue latéralement - sûrement un tribunal, d'après la scène qui s'y joue. Au centre, de profil, un gamin, douze ans au plus, mal attifé, pull trop large pas très propre, chaussures en sale état, casquette de cheminot de guingois sur une tignasse rebelle, un pansement au front; il se tient droit, mais tête baissée, l'air dur faussement contrit - on le comprend à ses sourcils froncés -, les mains dans les poches, une cigarette sur l'oreille. À droite, face à lui, surélevé, derrière une tribune, un homme grand, maigre, costume sombre très strict, nœud papillon, petites lunettes cerclées, cheveux noirs très courts, rasés sur les côtés, raie au milieu parfaite - on dirait un gangster de Tintin en Amérique. Penché vers l'avant, il a l'air furieux, il vocifère (mâchoire carnassière, trois postillons en sortent), le doigt pointé droit sur le jeune accusé. Autour d'eux, une foule disparate, des visages pas toujours distincts, l'atmosphère est chaotique, houleuse. Certains semblent prendre la défense du gosse - il a des amis de son âge dans la salle, mieux vêtus, parfois -, le regardent apitoyés, tendent un poing menaçant vers le juge ; d'autres considèrent l'accusé avec curiosité, l'un d'eux brandit une énorme loupe qui grossit démesurément son œil gauche, un autre prend des notes avec application (langue sortie sur le côté de la bouche), etc.

3 Sous le dessin, un titre en capitales :

\section{La Bande dessinée devant ses juges}

4 Les traits de l'accusateur principal rappellent ceux du psychiatre Fredric Wertham, l'auteur de The Seduction of Innocent, ouvrage de 1954 qui stigmatisait l'influence 
désastreuse des comics sur les petits Américains, et dénonçait cette lecture comme un facteur irréfutable de propagation du crime et de la violence ${ }^{1}$. Wertham n'était pas un prédicateur réactionnaire illuminé, mais un savant renommé, familier des criminels mineurs (directeur d'un centre psychiatrique spécialisé en la matière), plusieurs fois appelé aussi comme expert devant des cours de justice. L'intervention de Wertham contre les comics est sans doute le premier contact direct (du moins le plus spectaculaire) entre les sciences humaines et la Bande dessinée; contact frontal, musclé, où l'objet du savoir est avant tout cible à détruire. Une première répartition des " discours sur » en découle (il y en aura d'autres), qui distingue les pro et les contra. On s'occupera moins des seconds, qui en général préfèrent lesilence méprisant ou l'allusion dédaigneuse. Le cas de Wertham est évidemment plus singulier : d'abord parce que son attaque constitue en ellemême une forme de légitimation (évidemment involontaire), dans la mesure où elle accorde à la Bande dessinée un pouvoir considérable, un charme vénéneux et fatal.

Mais il y a plus. Si les raisonnements du bon docteur sont bien faibles ${ }^{2}$, si les conséquences de ses attaques furent lourdes pour les éditeurs de comics, on voudrait pourtant ici lui reconnaître un mérite considérable. Il y a toujours une justesse, inattendue, mais très réelle, du réquisitoire, aussi haineux fût-il. Le procureur Pinard ne lit-il pas mieux Madame Bovary - dans un discours certes «bas de plafond»-que $\mathrm{M}^{\mathrm{e}}$ Sénard, l'avocat de Flaubert ${ }^{3}$ ? De même Wertham perçoit une vérité de la Bande dessinée - pas seulement des comics - quand il la dénonce comme une mauvaise fréquentation, un petit voyou, celui dont nousébauchions plus haut le portrait.Une allégorie, donc? En partie sans doute, tant il est vrai qu'on peut établir une analogie entre ses traits et les caractéristiques du neuvième art. Comme lui très jeune, dernier né des arts, et justement pas forcément admis à leur table; né de qui ?, on ne sait, petit bâtard (fait de mots et d'images) que ni la littérature ni la peinture ne reconnaissent - aucune reconnaissance, d'ailleurs, de personne; fils du peuple, fils des rues, pas celles des quartiers chics, ni des faubourgs artistes - modestes mais coquets -, mais celles des bas-fonds gris, le gris des pages de journal, sa demeure première, son berceau, coincé entre publicités et chiens écrasés. Comment serait-il bien élevé et instruit? Il est forcément sans manières, sans culture, sinon celle qu'il a glanée au hasard, loin de l'école. Forcément, il n'en fait qu'à sa tête, ni subversif ni rebelle, ce serait déjà reconnaitre l'autorité, juste irrévérencieux et ricaneur ${ }^{4}$. On le voit, l'allégorie fonctionne. N'empêche qu'elle ne suffit pas, trop simplificatrice. Elle se targue de figurer une essence (plus qu'hypothétique) de la Bande dessinée. Entre cette dernière et le Voyou, les relations sont plus complexes. Rappelons, s'il est besoin, qu'il représente d'abord l'image infamante, encombrante, dont les défenseurs du neuvième art cherchent à se débarrasser. Leur tâche primordiale fut pendant longtemps de combattre le préjugé qui l'associe automatiquement à l'enfance et l'assimile à un divertissement au mieux inoffensif et niais, au pire vicieux et pervers. Il fallut (il faut encore) prouver, œuvres à l'appui, que la Bande dessinée s'adressait à tous les publics, était apte à tous les sujets, et tous les tons. Mais le Voyou est un adversaire coriace, pareil au sparadrap du capitaine Haddock. Il ne se réduit pas à une simple calomnie lancée de l'extérieur. Il rôde à l'intérieur, dans les bandes dessinées mêmes, il les hante.

6 Hanter, c'est apparaître. Et les apparitions du Voyou ne manquent pas. Dès 1865, il emprunte les silhouettes des insupportables Max und Moritz de Wilhelm Busch, ancêtres des Katzenjammer Kids créés en1897 par Rudolph Dirks (les garnements de Pim Pam Poum, en version française). Ses masques ne cesseront de se multiplier, du fameux Yellow Kid 
(Richard Outcault)àBart Simpson (Matt Groening)-quiont en commun plus que la couleur. Il est chez lui dans l'univers sans adulte de Charlie Brown et des Peanuts (Charles M. Schultz), avec Mafalda (Quino) et ses amis, raisonneurs et têtus. Spirou (Franquin, entre autres auteurs) en wallon signifie écureuil mais aussi «jeune garçon facétieux », ce que sont aussi Quick et Flupke dessinés par Hergé. Le même Hergé ne parvient-il pas à dompter le petit monstre, à l'apprivoiser pour en faire un boy-scout impeccable, poli, généreux, tendre, Tintin, le héros sans âge aux traits juvéniles ${ }^{5}$ ? Mais voilà, chassez le diablotin par la porte, il revient par la fenêtre - et Hergé crée Abdallah... De même Little Nemo, grondé par ses parents à la fin de chaque planche, est sans doute encore trop sage pour le Voyou, qui pousse Winsor McCay à inventer Flip, l'insupportable ennemi-rival-ami, précoce fumeur de cigares. On n'en finirait pas d'énumérer les fripons et galopins de la Bande dessinée, tous pays et époques confondus.

De l'innocent au délinquant

Les mots de Wertham

Les enfants de onze ans ne lisent pas que des comics animaliers - que leurs parents le sachent ou non. [...] Ils assistent au spectacle du crime, de la délinquance et de la déviance sexuelle. [...] J'ai ressenti le besoin de devenir non seulement une sorte de détective à l'affût des racines de la délinquance de masse dans les temps modernes, mais aussi une sorte de conseiller de la défense pour les enfants condamnés et punis par les mêmes adultes qui leur avaient permis d'être trompés et tentés (p.13).

Nombre d'adultes estiment que les crimes décrits dans les comics sont si éloignés de la vie des enfants que ces derniers ne peuvent les considérer que comme des choses imaginaires ou fantastiques. Mais nous avons trouvé qu'il s'agissait là d'une grossière erreur. Les comics et la vie sont liés entre eux. [...] Les formes de délinquances jusquelà réservées aux adultes sont de plus en plus commises par des enfants et des adolescents (p.25).

Tous les enfants drogués et tous les enfants servant de relais dans les trafics de stupéfiants avec lesquels nous avons noué contact étaient des lecteurs invétérés de bandes dessinées (p.26).

Il existe un bon nombre de sombres officines où les enfants se réunissent, souvent dans les pièces du fond [back rooms], pour lire et acheter des comics d'occasion. [...] Dans certains endroits de nos villes, des hommesrôdent autour de ces magasins qui sont quelquefois de véritables foyers de prostitution enfantine. Il est évident que les comics préparent à merveille les petites filles.

Quant à la prostitution enfantine homosexuelle, en particulier chez les petits garçons, elle s'accompagne de vols et de violences. Or la réticence des enfants à toutes ces activités est érodée par les comic books (p.187).

7 «La belle affaire! Tous ces petits héros ne sont que reflets et appâts pour séduire de petits lecteurs. Inutile d'aller chercher le génie de la lampe... » Évidemment, l'explication sociologique n'est pas sans pertinence. Simplement, il faut y regarder de plus près. D'abord, on peut répondre que le public enfantin n'est pas forcément le seul recherché, notamment par des strips (comme le Yellow Kid, publié dans le New York World puis le New York Journal) qui paraissaient dans des journaux qui s'adressent à des lecteurs adultes, et que la destination enfantine est un trait propre essentiellement à la production francobelge de la première moitié (et plus) du $\mathrm{xx}^{\mathrm{e}}$ siècle $^{6}$. Ajoutons aussi que le Voyou est présent dans des œuvres mêmes qui revendiquent leur distance avec l'illustré pour enfants; il est ainsi une figure obligée de l'autobiographie, au premier plan dans les «portraits de l'auteur en enfant désobéissant » - il n'est que de relire Le Petit Christian de Blutch, ou bien des pages de Persépolis (Marjane Satrapi) ou de l'Ascension du Haut Mal (David B. $)^{7}$, trois exemples pris dans la masse. Par ailleurs, il apparaît que cette analyse sociologique présuppose souvent que l'enfant est le destinataire naturel des bandes 
dessinées; elle repose donc, en dernière instance, sur un argument ontologique: les récits en images seraient enfantins par essence. Pourquoi cela? Pour leur simplicité ? On ne voit pas qu'elle serait inévitable. Difficile d'être convaincu, quand bien même on pourrait admettre une fascination spécifique des enfants pour les images: les bandes dessinées ne sont pas seulement des « livres d'images ». Certes, le présupposé ontologique peut être évité, et l'explication historique se suffire à elle-même : la Bande dessinée serait marquée par les romans pour la jeunesse du siècle, dont elle prendrait la suite ${ }^{8}$. En somme, il n'y aurait là que pure contingence. Il nous semble qu'en les banalisant, telle analyse manque les affinités, profondes et secrètes, qui rapprochent le Voyou et le neuvième art, unis par tout un réseau de facteurs dont aucun ne semble prévaloir. Comme si le petit démon existait réellement, tapi entre les cases, et tirait lui-même les fils : hélant les petits lecteurs, inspirant les dessinateurs. Comme si c'était ce même mauvais génie puéril qui poussait à gribouiller dans les marges des cahiers pendant les cours, et à perdre des heures, plongé dans des histoires idiotes et "mal dessinées». Autrement dit, quel que soit le bout par lequel on la prenne, on trouvera toujours quelque chose d'enfantin dans la Bande dessinée. Cela ne signifie pas qu'elle soit un enfantillage : enfantin n'est pas synonyme d'« innocent » et « gentil »-, ce serait bien mal connaître les enfants.

8 Le Voyou serait alors non le symbole, ni le démon, mais le visage de la Bande dessinée, celui que lui a donné l'histoire. Le visage est apparence, pas toute la vérité, sans doute ne doit-on pas s'y fier aveuglément, mais il n'est pas non plus un simple masque que l'on peut retirer sans dommage. Surtout, il détermine considérablement les relations avec autrui. Ainsi, quiconque se préoccupe de Bande dessinée sera d'abord confronté à ces traits que nous avons décrits ceux d'un art non pas mineur, mais délinquant juvénile. Les plus grands auteurs, les meilleurs commentateurs sont peut-être ceux qui ne reculent pas devant le Voyou, et qui savent le prendre pour ce qu'il est, sans l'assimiler imperturbablement au « medium » (terme sur lequel on reviendra), sans non plus n'y voir qu'illusion mensongère à décaper au plus vite. Dans ce face-à-face naissent toute une série d'affects, aux conséquences capitales pour les orientations des discours et les postures de qui les tient. Les savoirs ne procèdent pas seulement de décisions épistémologiques dictées par la raison, ils reposent aussi sur des sentiments éprouvés envers leur objet. Le visage du neuvième art, juvénile, insolent, sans finesse, tel que nous l'avons mis en scène, permet alors de mieux comprendre la nature et la forme des réflexions qui l'environnent et l'examinent. Wertham le haïssait, rêvait son éradication; certains n'en ont cure, le méprisent ou s'en moquent. D'autres chercheront à lui faire regagner le droit chemin, lui trouver une famille, un logis. Il a aussi ses chauds partisans, ceux qui l'aiment "tel qu'il est ", se voulant ses semblables, ou ceux qui l'encouragent à étendre son empire, et pour cela empirer, se faire plus méchant et agressif. Il est temps d'examiner deplus près tous ces parleurs. À commencer par le dernier évoqué : le fan.

\section{Partie civile}

9 Fan: nous conservons le terme qui s'est imposéhistoriquement, etdésigne l'amateur passionné, non pas seulement de bandes dessinées, mais de tout secteur inclus dans ce qu'on nomma « culture de masse » ou « industrie culturelle »; à ce titre, il est l'une de ces figures sociales inventées par le $\mathrm{xx}^{\mathrm{e}}$ siècle, qui désigne et incarne de nouveaux types de 
rapport à l'art, passion sans mesures, fétichisme', culte, folie, mais aussi exigence extrême. Jeune - forcément -, plutôt adolescent, il est le semblable du Voyou son idole.

Pour ces pratiques et expressions méprisées ou simplement ignorées, c'est un personnage majeur, le premier relais, indispensable, qui leur donne une visibilité sociale, aussi mince soit-elle, par-delà la simple consommation. Ce sont les fans qui construisent le temple dont ils se font ensuite les gardiens. C'est encore plus vrai dans le domaine de la Bande dessinée. Au commencement était le fan : auteurs, théoriciens, savants, commentateurs, tous (ou du moins une très forte majorité) ont d'abord fait partie de la bande du Voyou. Ce qui ne veut pas dire qu'il faille le restreindre à un rôle éphémère dans une vie, une phase enfantine. Le fan est d'une autre trempe, il revendique un discours autonome, distinct de celui du savant - que souvent, d'ailleurs, il n'apprécie guère. Antiintellectualisme fatal - et régressif - des complices et admirateurs du Voyou ? Bien sûr. Mais l'hostilité procède aussi de la rivalité. Le fan parle, son discours possède un support propre, le fanzine ${ }^{10}$. Certes le fanzine est souvent d'abord un lieu de création oùle fan (futur) auteur fait ses premières armes, avant (peut-être) de changer de statut, et de quitter celui de l'amateur ; mais amateur veut dire aussi passionné, et le fanzine sait aussi privilégier l'étude et le commentaire des œuvres aimées. D'aucuns pourraient nier que les phrases du fan relèvent du savoir. Mais il y a bien un savoir de l'amateur, que ne sépare pas un infranchissable fossé de ceux des spécialistes. Sans doute en diffère-t-il quant aux protocoles, aux méthodes, et surtout (mais pas forcément) quant à l'usage qui en est fait. En somme, une différence de degré plus que de nature. Quel est ce savoir "amateur", dans le cas de la Bande dessinée? C'est d'abord une (extrême) érudition, une accumulation d'informations, mimétique de l'amoncellement d'albums du collectionneur que semble être fatalement le lecteur de Bande dessinée - celui qui, sa faiblesse avouée, est sommé de répondre à la question «t'en as combien? ", tant la quantité est la mesure première de cette passion. Il connaît par cœur toutes ses séries favorites, il a tout lu, tout retenu. D'autre part, ses connaissances peuvent être aussi d'ordre technique, servir de critère pour fonder l'appréciation d'un style. Telles ressources sont évidemment consacrées à rehausser l'objet d'amour, à légitimer la Bande dessinée comme art au sens plein du terme. Ce qu'on aperçoit là, c'est le fondement même de deux grands types d'approches sur la Bande dessinée : empirique et théorique. Double tranchant, donc, de la parole du fan : elle encourage et fortifie, on vient de le voir, celle du savant ; mais elle s'en méfie, et se réjouirait fort de lui imposer silence.

Par-delà le refus puéril de phrases trop austères et indigestes pour accompagner cet art «naiff et tout simple » qu'est la BD, telle hostilité manifeste un refus de la récupération par un discours officiel de pouvoir/savoir. Le fan prend alors le parti de la contre-culture, et interdit toute légitimation nécessairement pernicieuse et dangereuse. Pas question de regagner le droit chemin, c'est le Voyou qui est sur la bonne route. Telle est par exemple la position d'un Frank Miller, fan devenu idole, attaché à l'intact pouvoir de subversion des comics. Nous verrons que son sinistre « Yellow Bastard » est bien un devenir selon ses désirs du «Yellow Kid» d'Outcault. Pour confronter la position de Miller avec une autre, voisine mais divergente, on lira avec grand profit ses conversations avec le regretté Will Eisner, qui, sur l'autre versant, se fait héraut de la légitimation à travers l'assimilation de la Bande dessinée à la littérature ${ }^{11}$.

Le savant: avec ce second personnage, nous entrons dans le vif dusujet. Ses mots sont lestés du poids de l'autorité, elle-même assurée par toute une série de garanties institutionnelles (inscription dans une discipline établie, diplôme, statut social, etc.). Il 
peut adopter des postures très diverses, parler différentes langues, s'inscrire dans des démarches multiples. On ne manque pas de travaux de recensement et de classification, selon divers critères, des nombreuses approches au moyen desquelles on a abordé la Bande dessinée : on pourra ainsi consulter, par exemple, les numéros 8 , 9 , et 10 de la revue Critix où Pierre Huard envisage le sujet ${ }^{12}$ (et le problématise, examinant les avantages et limites de chaque approche) de manière fort détaillée, ou bien le Petit Critique illustré de la bande dessinée de Harry Morgan et ManuelHirtz, " guide des ouvrages de langue française [et, dans sa dernière version, anglaise] consacrés à la Bande Dessinée ${ }^{13}$ ». Luc Boltanski, en 1975, dans le premier numéro des Actes de la recherche en sciences sociales, avait mis en lumière « La constitution du champ de la BandeDessinée ${ }^{14}$ », un processus où l'apparition d'un corpus de commentaires universitaires joue un rôle non négligeable. Inutile, donc, de dresser une longue typologie de l'ensemble des discours qui ont constitué la Bande dessinée en objet de connaissance, et d'analyser, au cas par cas, leurs limites et effets respectifs. Revenons-en plutôt aux rapports qu'entretiennent les savants avec la Bande dessinée, et à ce qu'ils impliquent quant à leurs discours.

\section{Réquisitoires}

On l'a vu plus haut, le savant est, historiquement, le négatif du fan, son ennemi mortel. The villain. L'amour de celui-ci pour la Bande dessinée n'aurait d'égale que la répulsion de celui-là. Le savant est (presque) toujours fou, fou dangereux. Les lecteurs de BD sont bien avertis. Wertham comme figure emblématique donc, et avec lui tous ces psychologues (certains professionnels, d'autres plus amateurs) des années 1940 et 1950 qui craignent l'influence pernicieuse du Voyou. Ce rejet trouve un fondement théorique très puissant dans La Dialectique de la raison (Dialektik der Aufklärung) de Theodor Adorno et MaxHorkheimer, plus précisément au chapitre intitulé «La production industrielle des biens culturels ${ }^{15}$ ». Il n'y est jamais explicitement question de Bande dessinée, ou de comics (juste des dessins animés de Walt Disney), mais elle ne peut rester indemne sous le feu nourri des deux auteurs. Leur cible est en effet ce qu'ils nomment, les premiers, « industrie culturelle» (p. 139-176). Ils la fustigent comme instrument de la domination sociale : la standardisation des produits - qui caractérise l'industrie culturelle - favorise celle des citoyens-consommateurs, soumis à un véritable processus de domestication de la part du système (processus que ses produits n'ont d'autre fin que de représenter et d'imposer à tous les esprits). Le compositeur et musicologue Adorno s'attaque en priorité à la musique "de masse ${ }^{16}$ ", mais aussi au cinéma ou à la télévision : en chacune de ces cibles, ses assauts portent plus précisément sur la répétition, trait décisif de l'industrie culturelle. Dès lors, la Bande dessinée, sous la loi des "séries", abri des figures stéréotypées, ne pouvait échapper à sa vindicte, fût-elle implicite.

De fait, elle sera souvent envisagée, dans la lignée d'Adorno - quand bien même il ne serait pas invoqué explicitement comme modèle -, en fonction de ses effets idéologiques (presque toujours néfastes, cela va sans dire).Difficile de nier la pertinence de telle approche, quand l'on sait le rôle joué par les bandes dessinées dans toutes formes d'embrigadement, propagande ou simplement (mais la différence est parfois impalpable) pédagogie: que l'on songe au catholicisme dominant des publications franco-belges (Tintin publié dans Couurs vaillants), au patriotisme de nombreux comics, le Captain America en étant l'exemple le plus patent (tandis que Superman luttait, à l'origine, non pour la patrie, mais, dans un contexte de crise économique, en faveur des opprimés et des plus 
pauvres), ou aux bandes dessinées réalisées sous l'égide du parti communiste français, Pif le chien ou Rahan. Dans de tels cas (et dans bien d'autres où la fiction est absente, et où il s'agit ouvertement de simple publicité ou de propagande), la Bande dessinée est bien un medium au sens premier du terme, chargé de véhiculer docilement un « message » à elle extérieur. Ainsi peut-on à bon droit étudier les modes par lesquels sont mises en scène les valeurs ouvertement défendues par les récits dessinés. Mais le biais le plus fréquent, et qui nous intéresse ici, est celui de la démystification ${ }^{17}$ : plutôt que d'adopter une posture neutre, il s'agit de débusquer les effets les plus sournois, les implications pernicieuses de récits à l'apparente innocence. Le paradigme en est le fameux ouvrage de Ariel Dorfman et Armand Mattelart, Donald l'imposteur ou l'impérialisme raconté aux enfants ${ }^{18}$, qui rappelle les pages d'Adorno-Horkheimer sur les dessinsanimés du même Disney. D'autres travaux proposent souvent une étude des stéréotypes (sous la forme «la femme dans la Bande dessinée », «le monde musulman dans la Bande dessinée ${ }^{19}$ », etc.) que la BD ne manque pas d'ancrer dans les cerveaux malléables des (jeunes) lecteurs. Inutile d'insister sur la faiblesse de nombreux travaux, due aux condamnations prononcées a priori et à l'indifférence totale à la Bande dessinée dans sa concrétude ${ }^{20}$.

15 Les critiques d'Adorno sont encore aujourd'hui monnayées par les gardiens autoproclamés de la culture officielle, aux mérites fort variables et aux conceptions par ailleurs divergentes (parfois radicalement) de celles des penseurs francfortois. Alain Finkielkraut ${ }^{21}$ et Georges Steiner ${ }^{22}$ en seraient, sur des modes et à des degrés différents, de bons exemples. La continuité entre Adorno et les lamentos contemporains sur la fin de la grande culture réside dans la stigmatisation de la Bande dessinée (on s'en tient ici à son cas) comme avant-poste de la barbarie montante, ce dernier terme, assez vague quant à son contenu, valant surtout par ses connotations. Du Voyou au Barbare (via la figure plus contemporaine du "Sauvageon»), le pas est vite fait: il annonce le déclin de "la" civilisation, une régression non seulement psychologique, mais anthropologique. Distinguons pourtant Adorno de ses imitateurs ou continuateurs contemporains. Contrairement à ces derniers, il ne doit pas être accusé trop rapidement d'élitisme : éviter telle méprise, c'est tout le sens de la substitution d'« industrie culturelle » à " culture de masse $»^{23}$; elle montre bien qu'il ne s'agit pas de s'en prendre aux masses. Aux masses, il ne faut pas opposer l'élite, mais sans doute un sujet individuel qui seul saura être gagné par la négativité de l'œuvre.

Quoi qu'il en soit, si elle perdure encore aujourd'hui, la posture accusatrice parait cependant restreinte à certains individus circonscrits, et semble fort datée - encore que les retours de bâton ne soient jamais à exclure - comme l'est, du reste, en bien des points (mais non en tous) la théorie esthétique d'Adorno. Celle-ci reposait en effet sur une conception radicale de l'œuvre, absolument irréductible à la production culturelle, impropre à toute consommation : la jouissance esthétique est condamnée sans restriction comme assentiment, c'est-à-dire reddition, au système de la domination. L'œuvre authentique sera donc nécessairement d'avant-garde, toute en négation de la société moderne, brèche dans la domination en quoi celle-ci consiste, "promesse de bonheur " pour qui en fait l'expérience. Des propos guère de saison, on en conviendra, la notion d'« avant-garde » ayant pris un coup de vieux. D'autre part, la Bande dessinée, si l'on s'en tient à sa situation spécifique, a gagné une légitimité fragile mais réelle (par des moyens que l'on verra), qui la protège des anciennes rages. D'autres formes artistiques l'ont remplacée comme épouvantail pour les défenseurs de la civilisation - le rap et les jeux vidéo en tête. Notons cependant que, dans l'époque de tolérance esthétique intégrale que 
nous vivons (où tolérance est synonyme d'indistinction mercantile), toute critique un peu virulente des produits culturels est perçue comme malvenue (sinon réactionnaire) ; il est entendu que la « culture » est inoffensive, à quelques exceptions près, et que mieux vaut s'attaquer au vrai péril, les masses ${ }^{24}$ elles-mêmes. Ce qui, en dernière instance, est la démarche inverse de celle d'Adorno. Détail piquant, en notre ère d'indistinction, ce sont précisément des acteurs de l'« industrie culturelle » qui aujourd'hui empruntent (plus ou moins consciemment) des outils, concepts et raisonnements à l'école de Francfort. Preuve d'une limite importante d'Adorno, qui ne faisait que fort peu de cas de l'intelligence et de la résistance des consommateurs comme des producteurs, vaincus d'avance par le système. Preuve aussi que ses analyses conservent une puissance et une fraîcheur intactes en ce qu'elle mettent en évidence une logique industrielle. Ainsi Jean-Christophe Menu, auteur, cofondateur et figure tutélaire de la maison d'édition L'Association, entend-il, dans sa diatribe Plates-bandes ${ }^{25}$, accuser les différences entre les "œuvres» et les " produits ». Il s'acharne pour ce faire sur la "récupération » pratiquée par les éditeurs dominants économiquement (au détriment des éditeurs «indépendants» ou « alternatifs »), mise au service d'une «standardisation » massive, qui sert les ambitions purement mercantiles de ces entreprises culturelles. Si la référence n'est pas explicite, et les concepts sans doute passés dans un usage assez courant, on entend dans le raisonnement d'incontestables accents adorniens - d'autant plus que J.-C. Menu en appelle à une « avant-garde » de la Bande dessinée.

La théorie et ses suites

Ou encore: nous devons passer par l'implacable réquisitoire d'Adorno et Horkheimer si nous voulons avancer une catégorie comme celle de l'œuvre en Bande dessinée. L'accusation garde ainsi sa validité dès lors qu'on déplace la ligne - et que la Kulturindustrie n'est plus prise en bloc. Il y a manifestement un échec de la pensée d'Adorno et Horkheimer sur ce sujet. Les deux auteurs ont trop cru dans l'efficace de la réclame. Considérer toute l'industrie de la culture d'un seul tenant est en porte à faux avec le mouvement de pensée qu'est la dialectique négative (qui s'invente progressivement au cours de la Dialektik der Aufklärung). Comme si l'innocence des deux philosophes se révélait à la faveur d'une séduction sur eux exercée. Indéniablement existe dans les magazines, les films, les émissions de radio et télé, un gigantesque et terrassant appel à la conformité. Or, cette proclamation se fait d'autant plus tonitruante qu'elle compte faire taire non seulement la critique externe, mais aussi la déviance en son sein. Le totalitarisme médiatique veut interdire la formulation de sa possible non-identité. Le verbiage de l'industrie doit être également dénoncé comme une censure préalable des marginaux. Le refus de l'œuvre de Bande dessinée est au fond une conclusion qui rassure la théorie critique comme les ronronnantes usines qui fabriquent de la bédé, de l'entertainment-comics, du manga à la chaîne.

Séduction malgré tout. À la fin du chapitre sur la Kulturindustrie figure la mention «(fortzusetzen)» (p. 198) - «(à suivre) ». Par un effet de contamination discrète, le mot supplémentaire après une phrase définitive sur « le triomphe de la réclame » ( ibid.) reprend le procédé des feuilletons radiophoniques et autres séries dessinées. Serait-elle ironique, cette étrange addition s'inscrirait encore dans la puissance maléfique de l'humour dû à l'industrie culturelle (voir p.167-169). Horkheimer et Adorno désignent peut-être ici l'incapacité à se soustraire intégralement du contemporain de masse. À moins que, sans le chercher vraiment, ils ne nous indiquent le dépassement d'une logique industrielle dans la traversée du pire. Le consommateur a les moyens de se transformer en amateur - au sens fort que nous développons dans cet article. L'auteur de Bande dessinée et son lecteur, contre toute attente (celle du savant séduit sans doute, celle du capitalisme culturel), peut transpercer la «standardisation » et le « normé » (p.183). 
Citations tirées de Max Horkheimer et Theodor Adorno, Dialektik der Aufklärung.

Philosophische Fragmente, Amsterdam, Querido, 1947.

\section{La Défense}

\section{Experts}

17 même que l'hostilité ne représente en fait qu'une phase historique et un courant minoritaire dans la bibliographie des travaux scientifiques sur la Bande dessinée. Passé les années 1970 (et la mode de «l'analyse idéologique»), le discours accusateur se fait plus rare, les contempteurs de la Bande dessinée préférant en général le silence indifférent. Les parleurs l'aiment, ou du moins le déclarent. Boltanski l'avait justement remarqué, la constitution d'un corpus de commentaires et d'études est avant tout celle d'un appareil de légitimation, qui confère une nouvelle dignité à l'objet jusque-là à l'écart des productions culturelles reconnues et fréquentables. On ne s'étonnera pas alors que les deux grandes catégories de discours savant/aimant recoupent le partage qui ordonnait les paroles du fan: l'histoire correspond à l'érudition, la théorie aux essais de délimitation de l'objet aimé. Sans doute la relation qu'ils entretiennent avec le fandom estelle asymétrique, la première en étant nettement plus proche que la seconde, on détaillera en quoi plus loin. Voyons-y d'ores et déjà deux manières de réhabiliter notre Voyou: lui offrir une famille, une généalogie décente, voire prestigieuse et lointaine (dans la peinture, la littérature) ; lui donner une identité qui lui permette d'être reconnu et accepté. Mais avant d'explorer les différentes formes de réflexion que recouvrent ces catégories, il faut regarder de plus près la « dignité » que le verbe savant conférerait à son objet, ainsi que «l'amour » que lui portent ces doctes témoins de la défense. L'une et l'autre vont se révéler fort ambivalents.

18 Le processus de légitimation auquel contribuent les savants devrait logiquement conduire à l'autonomie de la Bande dessinée, comme forme d'art (ou, si l'on préfère "pratique culturelle » qui ne tire pas sa valeur d'autres discours ou pratiques). Tel n'est pas toujours le cas, au point qu'il faut compliquer la dichotomie histoire/théorie d'une seconde distinction: une part des travaux savants (de l'historien, du théoricien) tendra vers un pôle autonome, une autre vers un pôle "hétéronome ». Hétéronome, le discours qui travaille à sa propre légitimation plus qu'à celle de son objet. Celui qui le tient s'intéresse au Voyou, certes, mais comme à un cas nouveau, fascinant, qui va lui permettre d'étendre son champ de recherches, de tester de nouvelles hypothèses, d'en valider d'anciennes. L'«amour» de la Bande dessinée qu'il clame bien haut tient plus de la convoitise narcissique du dragueur brûlant qui veut ajouter une nouvelle conquête à la liste de ses proies. Cela sans préjuger de la qualité des travaux en question. L'article de Luc Boltanski, maintes fois cité ici (et que l'on rangera dans les travaux d'historiens, une histoire ici équipée de pied en cap par la sociologie bourdieusienne), serait un bon exemple de telle posture, comme en témoignent ses lignes introductives ${ }^{26}$ :

La description des transformations qui ont affecté la Bande dessinée peut être l'occasion d'analyser certains mécanismes qui accompagnent l'apparition d'un champ formé sur le modèle des champs de culture savante. (C'est nous qui soulignons.)

Il s'agit bien de saisir une «occasion » pour l'analyse, non de s'intéresser à la Bande dessinée en tant que telle, et pour elle-même. Ainsi l'histoire culturelle se penchera 
volontiers sur la BD comme document, au même titre que n'importe quelle manifestation d'une « culture » entendue au sens le plus large du terme.

Sur le versant théorique, la sémiologie - «forme vulgarisée » du structuralisme et facteur de «relativisme culturel» selon Boltanski (art. cit. p. 42)-a certainement permis l'accession de la Bande dessinée aurang d'objet académique. Mais, quoiqu'elle se démultiplie en enquêtes régionales, la sémiologie, générale dans son projet même (et postulée comme telle par Saussure dans le Cours de linguistique générale), tend bien souvent - combien de fois ne le lui a-t-on pas reproché! - à ignorer la singularité des objets qu'elle appréhende avec des concepts issus de la linguistique, matrice historique plutôt que branche théorique du discours sémiologique. Ainsi les analyses ponctuelles d'Umberto $\mathrm{Eco}^{27}$, ou le brillant (et non moins ponctuel) ouvrage d'Alain Rey Les Spectres de la bande ${ }^{28}$ laissent-ils deviner l'amusement légèrement condescendant du grand savant s'encanaillant : il y a là, comme parfois dans Les Mythologies de Barthes ${ }^{29}$, ouvrage séminal en la matière, la démonstration d'une virtuosité intellectuelle, qui n'exclut pas la pertinence et l'intérêt de toutes ces analyses, mais marque une certaine distance avec son objet. Distance: le même terme, au prix d'une réélaboration conceptuelle, est posé comme garant de la scientificité même du discours. Elle n'est plus alors affectation inhérente à la supériorité du grand savant facétieux, mais posture nécessaire au travail scientifique d'objectivation, qui suppose le point de vue adéquat. C'est ainsi que Pierre Bourdieu prescrit la suspension de ce qu'il nomme illusio, soit la «croyance collective dans le jeu et dans la valeur sacrée de ses enjeux ${ }^{30} »$.

\section{Culture BD?}

Les plus récentes cultural studies changent-elles la donne? Rien n'est moins sûr. D'abord, contrairement à ce qui se dit ou se croit souvent, les comics ne sont pas forcément au centre de cette pluridiscipline. Aucun desquarante articles de l'énorme ouvrage de référence Cultural Studies publié en 1992 chez Routledge ne se consacre exclusivement aux bandes dessinées. Et les références ponctuelles demeurent allusives, anecdotiques. Mais le problème théorique réside davantage dans la fusion programmée des comics dans l'ensemble culture. La dénonciation adornienne est passée; lacollusion reste. Le telos des cultures et identités des personnes et des groupes n'a que faire de la potentielle spécificité des comics. Il ne s'agit que d'études de cas par le moyen de...

Partant, même l'intitulé Comic Book Culture renvoie à tout autre chose qu'une œuvre et désigne la fumeuse identité collective des fans. La Bande dessinée n'est sauvée par le chercheur qu'au prix de son utilité sociale désormais proclamée : "Peut-être la culture des comics apprendra-t-elle aux Américains comment réintégrer la vie culturelle dans un tout actif et participatif » (derniers mots du livre de Matthew Pustz).

Coll., Cultural Studies, Londres, Routledge, 1992. Matthew Pustz, Comic Book Culture. Fanboys and True Believers, Jackson, University Press of Mississippi, 1999.

Qu'elle soit une conséquence accessoire ou une composante nécessaire du discours, la distance est le point commun de toutes les démarches évoquées dans les lignes qui précèdent - où le savant est, plutôt qu'un témoin, l'expert étranger au procès, appelé à la barre par la défense pour ses compétences reconnues, non sans sympathie pour l'accusé, en général, mais guère plus. La Bande dessinée est par lui envisagée de l'extérieur, et s'il lui confère une valeur nouvelle, c'est à la manière du roi Midas : c'est de son don (ou " compétence ») que l'or jaillit, et tout ce qu'il touche se métamorphose de la même façon, y perdant au passage, s'il y a lieu, la vie. 


\section{Avocats} passion anime les doctes travaux, défenseurs acharnés et éternels de la Bande dessinée. Ils se donnent pour mission première de sauver de l'oubli leurs lectures enfantines, de constituer une mémoire collective de la Bande dessinée, d'en établir l'histoire. Notons au passage l'asymétrie que nous évoquions plus haut: la théorie n'émergera que dans un second temps, alors qu'elle est d'ores et déjà intégrée au champ académique, qui l'arme conceptuellement. Le discours historique, en revanche, se manifeste d'abord en marge de celui-ci, à travers des institutions entièrement consacrées à la Bande dessinée et à la construction d'une mémoire collective. La constitution d'une mémoire représente une première étape indispensable vers l'autonomie, qui donne visibilité à des œuvres oubliées, permet d'établir des lignées, et pose l'idée même d'une évolution des formes. Les clubs de Bande dessinée, sont, comme dans le domaine du cinéma (mais d'une visibilité sociale bien moindre), les acteurs de cette entreprise patrimoniale ${ }^{31}$. Ils sont à l'origine, en France, des premières revues d'étude (Giff Wiff pour le Celeg, Phénix pour la Socerlid), d'événements (la fameuse exposition de 1967, Bande dessinée et figuration narrative, organisée par la Socerlid), et de publications variées (le catalogue de l'exposition, par exemple ${ }^{32}$ ).

Les discours produits en ces différents lieux sont caractérisés par deux traits propres au fan : la nostalgie et l'accumulation. La première, flagrante, l'emporte bien souvent, dans les innombrables dictionnaires ou encyclopédies (qui perdurent encore aujourd'hui), sur les considérations épistémologiques. La sélection s'effectue selon les préférences personnelles plutôt que sur des critères objectifs. D'autre part, privilégier les entrées par personnages (ou, accessoirement, par genres ou séries), c'est employer les principes qui structurent aussi les collections. Sans doute devrait-on parler alors d'archivistes amateurs, et non d'historiens; de fait, nombreux sont les "spécialistes " de Bande dessinée autoproclamés, tant il est facile de s'ériger en spécialiste dans un champ où la validation du savoir n'est pas du tout institutionnalisée, ce qui est le cas à l'époque évoquée (les années 1960) et à bien des égards encore aujourd'hui. Cela ne veut pas dire que tous les ouvrages issus de ces recherches soient sans rigueur: au contraire, sur le même terreau fleurissent aussi des études proches de l'histoire de l'art, qui rapprochent la Bande dessinée de la peinture ou de la littérature. Les méthodes de telles études relèvent le plus souvent de l'histoire de l'art. Elles rencontrent de ce fait les difficultés propres à ce domaine de recherche : comment articuler l'histoire « interne », celle de la Bande dessinée, et le « contexte » ? Sur quels critères établir continuités et discontinuités, distinguer les séquences signifiantes, identifier courants, « écoles », mouvements, et leurs évolutions? Pour preuve de l'ampleur de ces obstacles, l'absence d'« histoire générale de la Bande dessinée » qui fasse référence (fût-ce pour être contestée ${ }^{33}$ ) alors que se multiplient les études approfondies cantonnées à une époque, une école, une forme, une zone géographique.

Surtout, ces travaux sont indissociables de la réflexion théorique: toutes les interrogations auxquelles se heurte l'historien mettent en jeu le problème de la définition. Inversement, la théorie de la Bande dessinée ne peut se faire à moins d'un effort d'historicisation, tant son objet semble défini par un contexte socio-historique précis. Il est rare d'ailleurs de ne pas rencontrer de digressions historiennes dans les ouvrages de théorie, et, à l'inverse, des développements théoriques (c'est-à-dire presque 
toujours le débat sur la définition) dans des études historiques. C'est ainsi que les deux débats les plus récurrents en matière de Bande dessinée concernent respectivement la définition et l'origine, deux problématiques nécessairement entrelacées ${ }^{34}$ et qui supposent des jeux d'aller-retour entre histoire et théorie, chacune s'efforçant de prendre le meilleur sur l'autre, mais l'une n'allant jamais sans l'autre - Dupond et Dupont de la recherche. Où se rejoue la confrontation entre histoire littéraire et poétique, et, plus généralement, entre histoire de l'art et esthétique.

Sans trancher entre les deux démarches, il faut revenir une nouvelle fois sur la nette différence de statut. Les ouvrages d'histoire de la Bande dessinée balancent entre le travail amateur et l'application locale de méthodes forgées et éprouvées dans le champ académique; la théorie de la Bande dessinée représente en revanche une tentative d'élaboration d'un savoir adéquat à son objet, car émanant de lui. Son ambition est de décrire le plus rigoureusement possible la Bande dessinée, c'est-à-dire d'énumérer, désigner et analyser les différents éléments qui la composent et leurs modes d'articulation; au cœur de ce projet, la mise en évidence de la fameuse spécificité. Le discours produit n'est alors pas directement en concurrence avec les autres, il leur est transversal.

Des lectures naïve ou savante, politique, sociologique, philosophique ou psychanalytique, aucune, a priori, ne doit être interdite. [...] Une précaution s'impose toutefois. Nulle lecture n'est à rejeter, à condition qu'elle ne méconnaisse pas totalement les particularités de son objet. [...] Contribuer à la mise au jour de ces mécanismes fondamentaux, tel est le premier objet des pages qui vont suivre ${ }^{35}$.

Ces phrases de Benoît Peeters (scénariste et théoricien) circonscrivent bien la place qu'entend occuper la théorie : celle d'un organon pour toutes les démarches qui tentent d'appréhender la Bande dessinée, un savoir fondamental qu'il faut maîtriser nécessairement. Il est d'ailleurs notable, en premier lieu, que des «praticiens » de la Bande dessinée se soient eux-mêmes illustrés dans ce domaine (plutôt que dans celui de l'histoire), tel Will Eisner, pionnier de la légitimation, auteur d'une œuvre considérable (tant par son ampleur que par son importance), qui a notamment proposé la catégorie d'« art séquentiel» (Comics \& Sequential Art) et a préciséses conceptions dans Storytelling ${ }^{36}$.

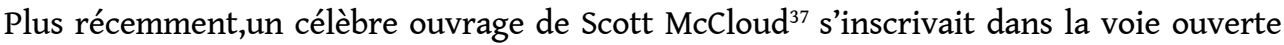
par Eisner, tâchant de circonscrire le territoire de la Bande dessinée et d'envisager ses possibilités, mais cette fois sous la forme même d'une Bande dessinée, signe d'un degré supplémentaire atteint dans l'ordre de l'autonomie ${ }^{38}$. Si elles sont remarquables, ces interventions d'auteurs dans la théorie restent néanmoins exceptionnelles, et les travaux sont menés pour l'essentiel par des chercheurs qui font de la Bande dessinée l'objet central - et non plus un détour occasionnel et marginal - de leur travail. L'évolution des recherches théoriques depuis les années 1970 peut se lire de plusieurs manières : d'abord comme un affranchissement progressif (et relatif) à l'égard de la sémiologie (informée par la linguistique structuraliste) et plus largement de l'Université. L'ouvrage inaugural en la matière de Pierre Fresnault-Deruelle, La Bande dessinée, l'univers et les techniques de quelques comics d'expression française ${ }^{39}$, s'inscrivait explicitement dans les études sémiologiques (il est sous-titré « essai d'analyse sémiotique »), tout comme, plus tardivement, les ouvrages de Pierre Masson (Lire la Bande dessinéé ${ }^{40}$ ). Les générations suivantes de théoriciens, en revanche, prennent leurs distances avec la sémiologie, jugée coupable d'une trop grande dépendance envers la linguistique et donc inapte à appréhender la spécificité de la Bande dessinée. C'est le cas, à des degrés divers et dans des perspectives différentes, de Thierry Smolderen, Benoît Peeters, Thierry Groensteen (auteur d'un Système de la Bande dessinée ${ }^{41}$ ) 
ou Harry Morgan (qui emploie un tiers de ses Principes des littératures dessinées à réfuter la validité de la linguistique structuraliste en tant qu'armature conceptuelle pour la théorie). Ce dernier en appelle même à une nouvelle discipline, la «stripologie " (le terme est attesté dès les années 1970), dont il ne fait que poser les bases (elle aura un axe majeur dans l'étude de la mimésis et ses modalités ${ }^{42}$ ). Même évoqué comme horizon plus ou moins lointain, l'avènement de la stripologie représenterait la consécration en terme d'autonomie, celle du discours savant parachevant celle de son objet. Notons cependant que l'éloignement de la sémiologie est lent, et relatif, puisqu'elle demeure pour ces savants la référence fondamentale, fût-elle négative ${ }^{43}$. Il faut ajouter qu'aucun des auteurs cités ci-dessus n'est universitaire, mais que tous sont inscrits de plain-pied dans le milieu de la Bande dessinée : Thierry Groensteen en tête, ancien rédacteur en chef des Cahiers de la Bande dessinée, ex-directeur du Centre national de la bande dessinée et de l'image, et fondateur des Éditions de l'An 2, qui publient la revue d'études $9^{e}$ Art. Puisque le statut disciplinaire de la théorie évolue, la question centrale qui l'anime, celle de la spécificité, se modifie en conséquence.

Ainsi l'alliance du texte et de l'image (avec sa variante, l'usage du phylactère) n'apparaît plus comme le critère définitoire, ce qui fut longtemps le cas. Selon le principe de balancier déjà évoqué, la théorie est sommée de tenir compte de l'historicité de son objet, et les théoriciens les plus récents franchissent allégrement la frontière poreuse qui sépare les deux disciplines ${ }^{44}$. Mieux, c'est en fait l'idée même de définition qui est mise en question: Morgan, cohérent avec sa virulente critique de l'impérialisme linguistique, considère ainsi qu'«il est prématuré de chercher une définition scientifique de la littérature dessinée » et se contente d'une " perspective descriptivo-interprétative » qui lui permet de mettre en avant les critères (temporaires) de "dispositif» et de « séquentialité ». Groensteen quant à lui n'est pas moins sceptique quant à l'identification positive de la spécificité, et préfère parler d'une condition nécessaire, la «solidarité iconique ». À côté du problème central de la définition, avec lequel il ne se confond néanmoins pas, l'effort théorique, à l'aide des concepts qu'il a produits, a permis une connaissance plus vaste et précise des possibilités de la Bande dessinée ${ }^{45}$.

On le voit, le champ de recherche est vivant, les débats y sont animés, et l'autonomie en est plus que jamais l'horizon et le fondement. D'où vient alors ce sentiment de «pénible surplace » que confie Jan Baetens ${ }^{46}$ ? Il évoque l'échec (jusqu'à maintenant) du projet de fonder une nouvelle discipline, la légitimation toujours partielle de la Bande dessinée, et identifie en dernière instance «la dérive formaliste, héritage de l'ancienne sémiotique des années soixante »; il prescrit une cure, selon lui nécessairement revigorante, de cultural studies (voir supra à propos de celles-ci). Encore le jeu du balancier, certes profitable, et source de progrès, mais sans doute pas totalement satisfaisant. On voudrait même voir l'une des causes de ce "surplace" déclaré par Baetens en son caractère exclusif, noué autour de la sempiternelle « spécificité ». Sans doute est-ce cette charnière qu'il convient d'observer d'un autre œil.

\section{Pour une critique de la Bande dessinée}

\section{Contre le medium}

Le statut même de la "spécificité " est en effet rarement objet de débats chez les chercheurs. La comparaison permettra d'y voir plus clair: malgré les apparences, la 
spécificité de la Bande dessinée n'est pas homologue à la littérarité que cherchent à identifier les théoriciens de la littérature (elle n'a d'ailleurs nul nom de baptême idoine). Cette dernière est pourtant elle aussi une "spécificité », celle d'un usage particulier de la langue, mis en œuvre par certains textes. Or, celle qui occupe les stripologues n'est pas propre à certaines productions, elle est celle du medium. Le mot est lâché. Omniprésent, jusqu'à la nausée, dans toutes les études et recherches concernant la Bande dessinée. Et pas simplement les travaux théoriques. Ce problème de la spécificité, qui se pose au premier chef aux théoriciens, fait affleurer dans leur champ une faille qui en réalité traverse et fissure l'ensemble des domaines de recherche examinés jusqu'ici. Poursuivons le parallèle engagé : le medium de la littérature est le langage verbal. Or, la théorie littéraire, même si elle a largement emprunté à la linguistique, ne s'est jamais confondue avec elle, tout simplement parce qu'elle n'est pas réflexion sur un medium, mais sur un art - quand bien même cet art serait censé révéler une essence dudit medium.

Touchant la Bande dessinée, le mot «art» est attesté pourtant, affecté de l'ordinal «neuvième». Pourquoi alors le terme «medium» conserve-t-il la préférence des commentateurs? Peut-être parce que « art » est trop empreint de culture légitime, qu'il dresse une haie de barbelés autour du territoire en question, qu'il enferme la Bande dessinée dans le rôle de petite sœur des arts consacrés. Sans doute y a-t-il de cela. Mais il y a plus, puisque même ceux qui n'ont pas ces répugnances semblent en rester à la perspective du medium: Scott McCloud dit vouloir envisager la Bande dessinée « en tant qu'art ", mais c'est le sens de technique qu'il faut donner au mot lorsqu'on lit L'Art invisible. Il en va de même dans les analyses de Will Eisner, qui pourtant lutta pour faire reconnaître la Bande dessinée comme art. Rien d'illégitime bien sûr dans l'étude du medium, son histoire, sa définition, sa portée sociale, ses effets, sesformes, etc., et les connaissances produites sont indéniablement précieuses. Par ailleurs, les études théoriques, historiques ou autres prennent souvent soin d'évoquer des différences entre les productions en Bande dessinée, de distinguer les chefs-d'œuvre du tout-venant. Ces considérations relèvent cependant en général de la précaution oratoire, et n'infléchissent en rien la démarche adoptée. Manque le discours qui fasse le départ entre différentes manifestations du medium, qui dise que telle Bande dessinée est une œuvre, et telle autre non, et pourquoi. Manque ce geste chirurgical, le coup de scalpel qui dissocie l'art et le medium. Tel geste porte un nom: la critique. L'opération séparatrice n'adu reste rien d'arbitraire, elle vient achever et mettre au jour la différence interne au medium (parfois imperceptible et secrète), celle qu'on appelle art, et qui existe dans et par les œuvres. On a insisté plus haut sur les sœurs ennemies histoire et théorie qui se disputent le monopole du savoir sur la Bande dessinée : cette configuration binaire est très particulière au sein des arts, où la critique est d'ordinaire première (l'on peut penser au cas du cinéma, si souvent associé à la Bande dessinée), tant et si bien que son nom finit par désigner l'ensemble divers des approches et méthodes d'analyse d'une forme artistique, comme dans l'expression «critique littéraire ${ }^{47}$ ». Dans le cas de la Bande dessinée un triple réflexe absolutiste a sans doute prévalu : celui du fan, dont la passion embrasse le medium dans son entier, et qui refuse toute amputation de l'objet adoré; celui de l'ennemi, qui, tout à sa haine, ne se préoccupe pas de séparer le bon grain de l'ivraie ; celui des savants, historien ou théoricien, qui font feu de tout bois, la question de la valeur n'étant pas pertinente sous le regard scientifique. Quoi qu'il en soit, la Bande dessinée est trop souvent, encore aujourd'hui, entendue comme un Tout, que l'on doit aimer ou rejeter entièrement. 
31 La critique, ce n'est pas le discours informatif et prescriptif, généralement journalistique - celui-là ne manque pas, aujourd'hui, à la Bande dessinée ; peut-être même n'est-il que trop présent. On entend par critique la mise en évidence qu'il y a œuvre (qui n'est pas simple appréciation subjective) à travers une confrontation directe. Le critique est le seul à considérer le visage de la Bande dessinée, à regarder le Voyou dans les yeux. Pas un savoir à proprement parler donc, plutôt une pensée qui circule à travers les savoirs, qui les informe (on voit mal comment l'histoire ou la théorie pourraient se passer d'une critique, aussi élémentaire soit-elle) et les démet, figurant l'œuvre au point où elle échappe aux savoirs. C'est dire, alors, qu'elle n'appréhende pas l'œuvre comme un événement strictement interne au medium, qui n'ait d'autres effets que sur ce dernier une restriction de champ qui est bien souvent le fait de l'histoire de l'art, mais aussi des auteurs eux-mêmes, comme Eisner ou McCloud, préoccupés avant tout, dans leurs réflexions théoriques, de l'évolution du medium, mais finalement assez peu de ses effets et des significations qu'il véhicule.

Nulle découverte ici: la quasi-absence d'une critique de Bande dessinée a été diagnostiquée à plusieurs reprises (du moins dans le champ français, notre référence privilégiée) depuis les années 1970 : on en trouvera une liste commentée dans « La théorie du zéro pour cent », de Jean-Philippe Martin, un article paru dans le premier numéro de la revue Critix (automne 1996), qui constitue elle-même l'une de ces rares tentatives de développer une critique de la Bande dessinée. Quelques exemples des plus récentes: d'abord, la très imposante revue L'Éprouvette (àl'heure qu'il estàson deuxième numéro), publiée par L'Association, où la réflexion emprunte les formes et les tons les plus divers, en textes ou en bandes dessinées, sous la plume de commentateurs ou d'auteurs ${ }^{48}$. Cette critique d'auteur, la revue Comix Club (aux éditions Groinge) la tente depuis plusieurs années, sous une forme aventureuse et expérimentale, work in progress en perpétuelle auto-évaluation. Enfin Internet ne se révèle guère plus hospitalier que les autres supports, et tandis qu'y fleurissent les blogs d'auteurs, la critique n'y occupe qu'un espace limité : citons cependant le très remarquable site $\mathrm{Du} 9^{49}$, où s'invente une écriture critique originale à travers des textes généralement brefs mais toujours incisifs et attentifs à la singularité de l'œuvre qu'ils abordent (mais aussi à distinguer fermement œuvres et production courante).

D'autre part, le théoricien ou l'historien peuvent fort bien adopter une posture critique, au détour d'un raisonnement, alors qu'ils arrêtent un instant le cours de leur pensée devant une œuvre: ainsi de Benoît Peeters ou Thierry Groensteen, dont les démonstrations théoriques sont étayées par une foule d'exemples isolables comme fragments critiques. Aux États-Unis, le grand succès populaire des comics a longtemps (et paradoxalement) empêché une critique plus spécifique. Tout récemment pourtant s'est formée une revue de recherche universitaire sur la Bande dessinée, TextImage, qui est sans doute l'une des premières à consacrer ses pages au neuvième art sans le rapporter systématiquement à l'impérialisme ou à la culture populaire ${ }^{50}$. Cette publication en ligne, encore à la marge du champ du savoir outre-Atlantique, s'attache à construire une nouvelle scholarship, en alternant propositions théoriques et close readings. Toutefois, insistons-y, la critique demeure mal-aimée, négligée, fragile. C'est que, en plus des obstacles déjà évoqués plus haut, un rude adversaire se dresse devant elle : l'Auteur. 


\section{L'Auteur : apparitions}

34 L'Auteur se confond avec le Voyou, il en est le complice, l'alter ego, pire : il est hanté par lui. Mais nous allons trop vite. La notion même d'« auteur » de Bande dessinée est loin d'être évidente, d'abord parce qu'elle est consubstantielle au concept d'art, qui, pendant bien longtemps, semblait bien trop grave et sérieux pour la Bande dessinée. La traditionnelle division des tâches entre scénariste et dessinateur (encore plus segmentée dans l'industrie des comics américains, dotés d'un véritable générique, avec lettreur, encreur, coloriste, etc.) ne facilite pas non plus l'identification d'une individualité créatrice. Silhouette quasi-anonyme et toujours remplaçable dans une économie sérielle, plongé dans l'ombre du Personnage tout-puissant, l'Auteur ne doit pas trop attendre non plus des discours savants : la déclaration de sa mort - ressassée à l'envi dans ces années 1960-1970 (Barthes, Foucault, etc.) qui voient l'émergence et le développement des recherches sur la Bande dessinée - est bien souvent l'un de leur articles fondateurs. C'est du moins le cas des deux démarches dominantes : la sémiologie et l'histoire. La seconde, concédons-le, peut cependant se développer autour de l'auteur, en prenant la forme de la biographie, ce qu'elle fit à de nombreuses reprises. Hergé en fut le premier - et longtemps le seul - bénéficiaire, qui réussit à devenir aussi fameux que son illustre créature, cas rare (Tintin ne fut pas le Sherlock Holmes d'Hergé, qui lui-même n'est pas Arthur Conan Doyle). Une forme critique autre que la biographie aide grandement cet avènement de l'Auteur, j'ai nommé l'entretien. Numa Sadoul s'est ainsi fait une spécialité de ces dialogues (avec Hergé, bien sûr, mais aussi Tardi, Uderzo, Franquin, etc.), qui présentent l'interrogé comme détenteur d'un savoir acquis dans la pratique, lui reconnaissant par là une autorité. Ainsi, la silhouette de l'auteur de Bande dessinée, inexistante, fantomatique, puis floue et évanescente, parvient peu à peu à se préciser et à prendre consistance. Le mérite en revient pour l'essentiel aux principaux intéressés, mais ils ne se suffisent pas à eux-mêmes: un tiers est nécessaire, ne fût-ce que pour donner la parole, et tendre l'oreille. Boltanski voyait sans doute dans cette constitution du "producteur» en " auteur » un processus calqué sur les champs culturels "savants ». L'homologie est bien là, mais il semble qu'elle soit déterminée plus essentiellement par une solidarité très puissante qui tient ensemble une série de concepts : art, œuvre, auteur, critique. Pas d'art sans œuvre, pas d'œuvre qui ne postule un auteur (celui-ci n'étant pas antérieur, mais coextensif à l'œuvre ${ }^{51}$ ), et pas d'auteur sans reconnaissance critique. L'auteur doit son existence même au critique. Pourtant (justement?) il ne l'aime pas. Du reste, plus généralement, il n'apprécie guère les discours savants qui viennent miner son autorité. Mais seul le critique est aussi vulnérable à ses assauts, qui n'a d'autre fin que de mettre au jour les œuvres et auteurs. Pourquoi tant de haine, alors? C'est que l'Auteur entend s'affranchir de la parole du critique, en assumer lui-même les tâches. 


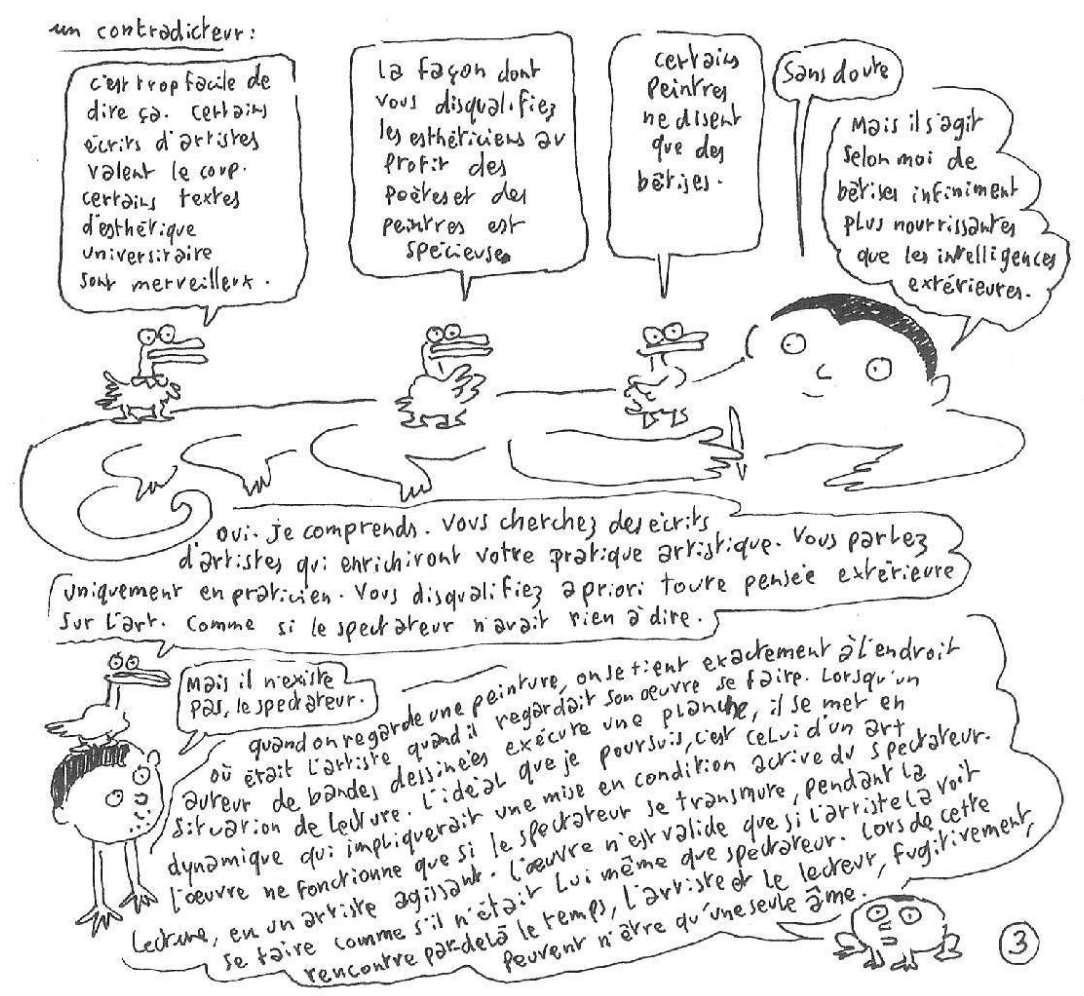

\section{L'Éprouvette $n^{\circ} 1$, Joann Sfar@ L'Association.}

Un texte de Joann Sfar, paru dans le premier numéro de L'Éprouvette ${ }^{52}$, illustre au mieux cette hostilitéde l'Auteuràl'endroit du critique.On y retrouve les griefs classiques contre la critique, le parasitisme, l'absence de légitimité, l'appropriation pernicieuse, le vampirisme même (et en matière de vampires, Sfar est expert) qui laisse les œuvres exsangues et sans vie. C'est que, plus essentiellement, Joann Sfar nie tout intercesseur entre Auteur et Lecteur, deux instances qui d'ailleurs se nourrissent l'une de l'autre, toujours en symbiose, en une dynamique de perpétuel échange des places qui n'admet aucun intrus ${ }^{53}$. Oui, un discours sur l'œuvre, distinct de celle-ci, est possible, mais alors seul l'auteur est à même de le tenir. Telle prise de position n'est pas ponctuelle et isolée chez Sfar, défenseur volubile, cultivé, et passionné d'un (neuvième) art - cette volubilité même, manifestée, phénomène encore rare dans la Bande dessinée, par les préfaces ou postfaces de ses ouvrages, est marque d'une posture d'artiste qui tranche avec l'humilité du praticien sans phrases - qu'il place aussi haut, voire plus, que les autres, admirateur éperdu des maitres qui l'ont inspiré, et l'inspirent encore. Mais plus fortement, son argumentation apparaît comme très cohérente avec toute son œuvre, comme sa doublure théorique (elle n'en découle ni ne la précède, doublure alors, plutôt que cause ou conséquence).

\section{L'Auteur : invention - sur Joann Sfar}

On pourrait en effet y lire - un choix parmi d'autres dans une matière riche et foisonnante - une défense et illustration de l'Auteur, par tous les moyens possibles, et d'abord, précisément, en refusant de se cantonner à une "Bande dessinée d'auteur " opposée à la production commerciale. Loin de tout éclectisme un peu creux (ou simple appât du gain), il s'agit justement de sortir l'Auteur du pré carré qu'on lui concède et 
d'asseoir son empire sur tout le territoire du neuvième art. Sfar s'empare ainsi des attributs traditionnels de la "Bédé " - autant d'obstacles, on l'a dit plus haut, à la reconnaissance d'un statut d'auteur - pour en retourner le pouvoir inhibant au profit de l'Auteur. D'abord, il balaie la dichotomie dessinateur/scénariste, et la spécialisation qui en découle, occupant indifféremment (voire simultanément) les deux fonctions (il n'est certes ni le premier ni le seul, mais c'est à noter). D'autre part, multiplier les séries comme il le fait - Le Chat du Rabbin ${ }^{54}$, Petit Vampire, Grand Vampire, Professeur Bell ${ }^{55}$, Les Olives noires ${ }^{56}$, Pascin ${ }^{57}$, etc.-sans jamais(jusqu'à maintenant) les conduire à leur terme, en ménageant entre elles toute une série de passerelles (thèmes, motifs, personnages récurrents), c'est en fait mettre en échec le principe sériel, et avec lui la tyrannie du personnage, et le soumettre à celui, plus puissant, d'une instance supérieure: la précellence s'inverse, ce ne sont plus les séries et les personnages qui voient passer différents auteurs (tels Spirou ou Lucky Luke, qui les collectionnent). Et pourtant c'est bien le cas dans Donjon ${ }^{58}$, la série aux quatre ramifications (" Zénith", «Potron-Minet ", "Crépuscule», «Monsters») dont Sfar et Lewis Trondheim sont les promoteurs et scénaristes, qui choisissent des dessinateurs pour une sous-série, ou même, comme le veut le principe de "Monsters", pour chaque album. Contrairement aux apparences, il s'agit là encore d'une exaltation de la figure auctoriale : les différents "Monsters » sont en effet pris en charge par des dessinateurs déjà adoubés comme auteurs (dont l'univers est généralement à mille lieues de l'heroic-fantasy), ou en passe de l'être (et, par un mécanisme d'entraînement, participer à «Donjon Monsters » est une étape importante vers la reconnaissance); la diversité stylistique et la "patte " personnelle imprimée à chaque album deviennent alors des traits caractéristiques de "Monsters", devenue, quantitativement, la série principale, et un symbole des conquêtes auteuristes.

Pour en rester à Sfar, il est évident que sa biographie romancée du peintre Jules Pascin ${ }^{59}$ constitue une pièce essentielle du dispositif, et à plusieurs titres. D'abord, parce que la Bande dessinée s'y saisit de la peinture, la grande sœur respectable et intimidante, à qui le Voyou fait perdre toute solennité en soulevant ses jupes. Ce portrait d'artiste, où il est autant question (voire plus) de sexe que de peinture, donne chair à l'auteur par le même geste qui démystifie le Grand Art et balaie insolemment les hiérarchies esthétiques. Surtout, Pascin s'inscrit dans une veine autobiographique implicite mais certaine: nul doute qu'il y ait beaucoup de Sfar en Pascin; or, la question est capitale pour ce qui nous occupe, c'est-à-dire la figuration de l'Auteur. Sfar clame souvent bien haut son peu de goût pour la Bande dessinée autobiographique. Il est dès lors facile de lui rire au nez en lui reprochant à la fois narcissisme et incohérence lorsqu'il publie ses carnets, ou même lorsque sa vie et sa personnalité sont évoquées de manière transparente à travers certains personnages (le Michel de Petit Vampire, par exemple). Facile, mais erroné. L'autobiographie est la forme la plus périlleuse pour qui construit un personnage d'Auteur, car elle présente toujours le risque que l'individu, cette autre personne, prenne le pas sur ce dernier. Or, l'ambition « auteuriste » de Sfar, dont témoigne Pascin (et qui n'a rien à voir avec la "prétention » ou la «mégalomanie », mais bien avec une conception exigeante de son art), requiert, un jour ou l'autre, la tentative autobiographique: la refuser, ce serait s'avouer vaincu, reconnaître implicitement l'irréductibilité de l'individu. Les Carnets sont la forme qui permet de soumettre l'Individu à l'Auteur : Sfar s'y met en scène, avec ses familles, ses amis, ses pensées et ses goûts, mais Joann, son personnage, est toujours avant tout dessinateur-écrivain, ou mieux, créateur toujours 
désireux (et le désir n'est pas un vain mot pour Sfar) d'exercer un art, pas forcément le neuvième, la musique, par exemple (Harmonica, 2002; Piano, 2003; Ukulélé, 200360).

On perçoit mieux, en cheminant en quête de l'Auteur dans l'œuvre sfarienne, la tension dynamique qui l'habite. Cette entreprise démiurgique (combien de fois, et avec quelle force Sfar n'aura-t-il pas évoqué son désir de " construire un univers ») est tiraillée entre deux pôles : d'un côté le goût immodéré des grands espaces, des histoires infinies, du mythe et de la fable, et de l'autre l'attention amoureuse pour la vie quotidienne, ses souffrances, joies et doutes, le besoin de la vivre pleinement, c'est-à-dire de la dessiner. Besogne d'Auteur, ce perpétuel va-et-vient entre dehors et dedans, repli et dilatation, tâche jamais achevée de celui qui crée et se crée en même temps. L'équilibre est forcément précaire, et de nouvelles combinaisons toujours à réinventer.

Restons un instant encore avec Sfar, pour un dernier trait d'auteur : l'affirmation, dans sa texture même, d'une parole auctoriale, autonome et légitime. L'article cité plus haut, comme tant d'autres écrits de Sfar, ses carnets, par exemple, est en caractères manuscrits, et non imprimés. Il n'est aucune solution de continuité entre les mots et les images : les premiers font partie du dessin, les secondes s'inscrivent dans le discours. Parler en tant qu'Auteur, c'est nécessairement faire appel à son art. On pourrait se passer du critique, alors ? Il suffirait de laisser s'exprimer le créateur, seul à même, dans le fond et par la forme, à dire le vrai de l'œuvre.

Tenons que non. D'abord parce que le verbe de l'Auteur, lorsqu'il se déclare en dehors de l'œuvre (comme expression d'un savoir spécifique, donc), n'est jamais aussi pur qu'il le souhaite, et puise en dernier recours dans les savoirs traditionnels déjà évoqués. Sfar fait ainsi usage de l'histoire de l'art et de l'esthétique, qui constituent le cœur de son propos, et le dispositif en lequel il les présente est avant tout un garant (auctor, en latin) de son statut. Dès lors, on peut se demander, si, hors de l'œuvre - car telle est bien la position que nous examinons ici -, l'Auteur est vraiment à même d'adopter une démarche de savoir qui lui soit propre, et, surtout, en quoi celle-ci pourrait prétendre à une plus grande légitimité. L'article de Sfar illustre ce qui advient dans la majorité des cas : Eisner expose des connaissances techniques, rassemblées et théorisées en une approche qui relève de la narratologie, et sous une forme qui n'est que fort peu «spécifique » à son objet. La bande dessinée théorique de McCloud semble pouvoir échapper à ce reproche : pourtant, le dispositif par lui conçu, tout brillant, riche et attrayant soit-il, n'est après tout qu'une " traduction » d'un classique traité théorique, qui aurait fort bien pu avoir un tout autre énonciateur, non "praticien». Certes, la distinction structurelle entre le propos démonstratif et les exemples est brouillée par l'usage de la Bande dessinée: le premier est exposé dans les phylactères du McCloud dessiné, les seconds étant totalement coextensifs au discours. Nous ne parlons que de "brouillage», cependant, car cette distinction existe toujours, qui définit la rhétorique argumentative la plus traditionnelle qui soit. L'exemple de McCloud, unanimement célébré comme inventeur d'une critique de la Bande dessinée en bande dessinée, montre bien les limites de cette défense et illustration de l'Auteur comme savant. Dès lors qu'il se déprend de l'influence du Voyou, il s'inscrit dans des champs et des méthodes bien identifiées. À moins qu'il ne se fasse critique. En apparence, nous rejoignons ici Sfar in extremis, pour qui « un texte sur l'art n'a d'intérêt que s'il constitue lui aussi une mise en danger poétique ${ }^{61} »$. On interprétera pourtant l'assertion au rebours dudit Sfar : il n'y a pas de discours spécifique de l'Auteur sur l'art ou sur l'œuvre (hors de cette dernière); s'il s'engage dans une démarche distincte 
des recherches savantes et au plus près des œuvres (les siennes ou celle des autres), alors il sera Critique.

\section{Fuvre et critique}

\section{Autoprésentations contemporaines}

41 Ainsi, le fait qu'un auteur de BD prenne la parole dans une revue ou une publication à lui consacrée ne signifie en rien qu'il échapperait par nature aux principales polarités qu'on a trouvées à l'instant. Même, on vient de le voir, certains dessinateurs et scénaristes se clivent, et vont prendre une position déjà connue, qu'ils répètent ou mobilisent stratégiquement. Il faut ici aussi abandonner le personnalisme diffus qui ferait de l'individu auteur un interprète privilégié de sa production. La rupture énonciative qui consiste à passer d'un registre à l'autre - de la Bande dessinée à l'à-plat du péritexte, en particulier - est si périlleuse qu'elle ferait dérailler les mieux intentionnés. Il suffirait de comparer, à la fin de l'édition en volume de From Hell, les longues et méticuleuses notes d'Alan Moore avec la drolatique historiographie dessinée des spécialistes de Jack l'Éventreur. Les notes éclairent l'ampleur de la documentation amassée par les deux créateurs pour la reconstitution de la Londres fin de siècle. En ce sens, elles fascinent, mais on sent un peu trop la volonté apologétique. Le besoin de justification - les comics ne sont pas le seul terrain des dilettantes - est écrasant; le martèlement référentiel (ceci est de la fiction, cela n'en est pas) catégorise, là où l'œuvre défait les contours du vrai et du faux. Par contraste, la postface écrite par Moore et dessinée par Eddie Campbell relance toute la narration. Les personnages apparus dans les multiples épisodes de From Hell réapparaissent, nouvelles marionnettes agitées par les théoriciens du crime en série. Le jeu de mots sur gull (le « vrai » nom de Jack selon la BD, et le mot anglais pour mouette) transforme les recherches sur l'Éventreur en grande chasse auvolatile. Les «ripperologues» se poursuivent sur un long rivage, qui signale la tâche infinie des chercheurs et rappelle la grève où se promènent le policier et le medium enquêteurs au début et à la fin du livre. Le contraste entre ces deux types d'épilogues est éloquent. Il permet d'insister sur le contenu de la proposition que nous avons formulée : il est peutêtre temps de faire de la critique, et de se concentrer sur ce que la Bande dessinée (et non la personne de ses créateurs) dit et montre d'elle-même.

Ce geste particulier sera nommé ici autoprésentation, et nous reviendrons sur les implications du préfixe. Juste avant cela, précisons. La BD se présente dans la BD. Elle se désigne comme discours et comme forme, agençant les traits qui permettent de la comprendre et de la lire. Et aussi elle renvoie à son apparition locale, à ce qu'elle est en train de faire ici et maintenant. Si l'on veut exploiter le sens social des présentations, un manga est à même de donner son nom de famille - son insertion dans une histoire et des pratiques, qualifiant la Bande dessinée en son entier - et ses prénoms - la distinction propre qu'il opère dans son clan, sa déclinaison d'identité, oùdéclinaison passe par tous les sens. Nous préférons auto-présentation àd'autres catégories, qui sembleront proches peutêtre, comme réflexivité ou spécularité. Ces mots recèlent une gênante métaphore optique, qui enracine la connaissance et la lecture dans la seule vision. Ils risquent, en outre, de trop accentuer l'identité et le préexistant. Car s'il est vrai que la Bande dessinée nous arrive aujourd'hui dotée d'une histoire, l'histoire d'une œuvre déplace tout le sens du connu. Il s'agit moins d'ouvrir une fenêtre ou de voir son reflet (fût-il déformé) que de 
rendre présente une nouvelle signification. La qualité de ladite présence, physique ou fantomatique, est une question seconde, qu'on laissera de côté pour l'instant.

À considérer les BD parues depuis dix ou vingt ans, l'autoprésentation semble une dimension indéniable, un fait presque envahissant. Les récits autobiographiques ou autofictifs, les carnets de voyages et journaux dessinés abondent. Courte sélection: le fameux et pionnier Maus d'Art Spiegelman évoque l'extermination des Juifs et la difficulté qu'a un fils de survivant à rendre la mémoire de l'expérience concentrationnaire, Will Eisner relate sa jeunesse de dessinateur dans The Dreamer, avec After the Snooter Eddie Campbell rend compte de son entrepriseéditoriale et existentielle, Jirô Taniguchi se livre à de subtiles variations mnésiques dans la tétralogie Quartier lointain, Marjane Satrapi s'inscrit dans une géopolitique du je grâce à Persépolis, Delisle évoque ses séjours de travail dans l'animation à Pyon-Yang. À quoi il faut ajouter des livres commentés, ou non, dans ce numéro, Approximativement de Lewis Trondheim, l'Ascension du Haut Mal de David B., ou le journal de Fabrice Neaud; et beaucoup d'autres encore. Mettant en scène un je dessinateur (effectivement, virtuellement), ces albums procèdent à l'inclusion directe du dessinateur scénariste dans les pages qu'il signe. Il arrive que cette littérale inscription laisse au fond peu de place à un discours explicite sur le fonctionnement de la saisie autobio-graphique (dans la liste précédente, c'est surtout le cas de Satrapi). Pourtant, même là, on prend position contre une tradition de la bédé d'évasion, d'où l'auteur voulait s'évader en refoulant le subjectif. Le dessinateur est indessinable dans Alix ou Blake et Mortimer, ou dans XIII et Thorgal; son officiel effacement est censé garantir la " qualité " de l'aventure. Au contraire, l'autoprésentation d'un (ou de plusieurs ${ }^{62}$ ) sujet(s) dessiné(s) redistribue la coupure entre la fiction et son auteur. De la sorte, la Bande dessinée intervient sur une longue tradition de censure (au nom de l'évasion), tout en exhibant l'histoire d'un processus créatif. Fidèle à la discontinuité consubstantielle à sa narration (la case, la planche, la page), la BD permet alors au lecteur de recomposer à son tour ce que le diariste ou la mémoire lui fournit.

À part l'assignation subjective, l'insistante apparition de l'homme-de-bande-dessinée comme personnage joue un rôle comparable. Doublant le narrateur, occulté ou pas, ce surnuméraire a d'abord une fonction proche de celle de la coccinelle de Gotlib : il signale, et, même muet, il commente. Il décale en outre le processus de véridiction traditionnelle que je viens de décrire. Le vieil auteur déchu de Box Office Poison (d'Alex Robinson), avec sa moumoute et son caractère atrabilaire passe dans la fabrique du personnage à un niveau plus latéral que par l'autofictif. Il incarne un hiatus interne à la création dessinée; le je, cette fois, s'est transféré un cran davantage, et avec lui la tenue unitaire du réel dans la BD.

Deux autres aspects dominants de l'autoprésentation contemporaine sont à retrouver dans la référence assumée à d'autres œuvres dessinées - et dans la glose. Cette dernière catégorie, dont les difficultés ont été soulignées, pourrait paraître marginale. Son importance n'est pourtant pas négligeable, surtout à un moment où - aux États-Unis surtout - la réédition en volumes de séries s'inspire de la réédition des films sur DVD, et inclut de plus en plus de «bonus». Pour citer un auteur dont un autre travail nous retiendra, David Mazzucchelli a récemment ajouté un singulier commentaire dessiné aux illustrations qu'il avait données à un scénario de Batman écrit par Frank Miller ${ }^{63}$. Mazzucchelli, tout en rééditant en appendice ses premières planches d'enfant (figurant le Dark Knight, évidemment), évoque son travail passé dans un style graphique bien différent des aventures qu'il commente, et, à l'occasion, reproduit la manière dite «operatic " de 
Frank Miller dessinateur. L'adoption d'une telle auto-présentation de l'œuvre actuelle par rapport aux dessins réalisés auparavant souligne le scepticisme affiché par Mazzucchelli sur sa propre réussite - et sur l'introduction du «réalisme» dans les comics de superhéros.

La référence à la $\mathrm{BD}$ comme construction (avec ses codes, ses tropismes, ses histoires...) est ainsi alliée à la glose. D'une manière générale, les quatre traits principaux de l'autoprésentation contemporaine sont à prendre comme autant de caractères compossibles, pour reprendre ce malheureux terme à la philosophie leibnizienne. In the Shadow of NoTowers d'Art Spiegelman concentre ces différentes tendances. On yoscille entre le je de Spiegelman, auteur de comics et New-Yorkais frappé par l'attentat contre les Twin Towers, et cet il du témoin de la catastrophe, mis à distance par la subjectivité créatrice. Les difficultés d'expression de la terreur sont reconnues, et déjouées par une tension entre les figurations anthropomorphique et zoomorphique (celle de Maus). Une autre solution se fait jour aussi, avec la représentation du je sous les traits des principaux personnages de comics du début $\mathrm{du} \mathrm{xx}^{\mathrm{e}}$ siècle. Les dernières pages incluent des reproductions de planches originales, précédées par un texte mentionnant les vieux comics comme seul réconfort (avec la poésie) dans une période traumatique.

\section{L'œuvre de la Bande dessinée}

47 S'il est indéniable que cette prise en charge de la BD par elle-même est très voyante aujourd'hui, deux questions surgissent concomitamment. Assistons-nous d'abord à une simple mode? Puis, que nous est-il présenté vraiment? La première interrogation mérite une remarque préalable. Quoique les œuvres concernées par cette «tendance » soient fort nombreuses, il semble que l'essentiel des comics de Marvel et DC ne soient pas des autobiographies, que les mangas de samouraïs et de science-fiction comprennent plus de références implicites que reconnues, et que les albums franco-belges à succès ne soient pas un lieu de gloses par excellence. Chaque point pourrait être vigoureusement contesté, mais admettons néanmoins. À reconnaître donc que le phénomène décrit n'est pas universel, on est amené à tirer deux conclusions provisoires. D'une part, toutes les bandes dessinées ne se valent peut-être pas; laissons ouvert l'énoncé pour plus tard. D'autre part, rien n'assure que les manières de l'autoprésentation s'épuisent dans les procédures que nous donnions tout à l'heure; l'accent contemporain peut nous aider à réinterpréter d'autres façons, éventuellement plus excentrées mais également signifiantes.

Il faudrait se défier d'une approche téléologique. Il est douteux que notre aujourd'hui dans la Bande dessinée doive correspondre naturellement à un stade de développement $\mathrm{du}$ " neuvième art ». Bien qu'il y ait maintenant beaucoup de trentenaires et plus parmi les accros de BD, cela n'a point pour corollaire que le medium soit enfin entré dans l'âge adulte - dont l'une des paradoxales manifestations, au passage, serait une exploration grandissante de l'enfance grâce à l'autobiographie. À l'inverse, ne croyons pas trop vite que le «narcissisme» soit la preuve de la dégénérescence d'un art pur et innocent, rattrapé par un intellectualisme nécrosant - dont le présent discours serait un stigmate supplémentaire. Ce dernier schéma, imité de celui de Nietzsche pour la tragédie grecque, est aussi inexact que son modèle. Ne manquons pas de le souligner. Les références que la $\mathrm{BD}$ fait à la BD sont peut-être plus difficiles à négliger aujourd'hui, mais ne datent pas d'hier. Au début des années 1960, les premiers numéros des Fantastic Four incluent des moments éloquents d'autoprésentation. Dans le deuxième épisode déjà, Reed Richards 
convainc le chef extraterrestre Skrull de ne pas envahir la terre grâce à de prétendus clichés de monstres. Le savant pense alors: «J'espère qu'il ne suspectera pas qu'ils sont en fait tirés de Strange Tales et Journey into Mystery ${ }^{64} »$ Le dénouement se fait donc ici par l'intermédiaire d'imagesreproduisant les publications Marvel. Un peu plus tard, dans le quatrième numéro, Johnny Storm retrouve Namor sous les traits d'un clochard amnésique, juste après avoir lu un vieil épisode de Sub-Mariner, datant des « fourties ${ }^{65}$ ». La publication du Sub-Mariner (dessiné, comme les Fantastiques, par Jack Kirby) avait alors cessé. Le retour d'un personnagese fait donc depuis les comics. Il y a un double plan intégral : ces aventures ne sont que de la Bande dessinée, et elles sont si réelles qu'elles n'occultent pas la présence même des comics dans cet univers narratif. Un épisode un peu plus tardif s'intéresse encore davantage à l'installation de ces deux plans et aux intersections possibles. En 1963, c'est le docteur Fatalis en personne qui vient rendre visite aux créateurs Stan Lee et Jack Kirby ${ }^{66}$. Tous deux sont présentés (ici et ailleurs) comme des sortes de reporters, rendant compte des gestes réels des Fantastiques. Pourtant, Fatalis les interrompt dans une séance de brainstorming, dont l'objet est l'invention d'un nouveau "super-vilain ». Lee et Kirby vont être forcés d'obéir à Fatalis, qui, par leur entremise, mettra une nouvelle fois en péril la vie des Quatre. À l'évidence, toutes ces scènes ont une fonction humoristique. Comme dans le théâtre comique, la rupture de la convention fictive provoque le rire - ainsi l'Avare de Molière désignant les coupables potentiels parmi les spectateurs. Mais ce désaccord interne n'empêche pas que l'action se poursuive, et, en l'occurrence, que les Fantastic Four révolutionnent les comics.

Il ne serait pas valable de réfuter ces exemples au nom d'une nostalgie plus grande encore pour la Bande dessinée de l'âge d'or, inconséquente, naïve et magique. En 1922, un journal tombe sur la tête de Krazy Kat, qui s'étonne de se trouver sur le papier en compagnie de son ami-ennemi Ignat $z^{67}$. Question effrayée posée par le chat à la souris : « Mais si je suis ici et que tu es là, comment se fait-il que je sois sur le papier et toi aussi - réponds donc à cela. » Encore plus avant dans le temps, au tout début, les séries de six cases de Desperate Desmond promettaient « un drame d'amour et de haine mis en images, avec une émotion forte dans chacune d'elles ${ }^{68}$ ». En plus du dessin et des phylactères, chaque case est accompagnée d'un commentaire verbal. Cette glose intégrée déconstruit ce qu'elle interprète, avec une opiniâtreté singulière. Dans l'un des épisodes, parus en 1910, alors que les deux personnages positifs n'ont toujours pas été inquiétés par l'horrible Desmond, une des gloses indique : "Quelque chose va se passer assez vite. C'est la cinquième image, et il n'en reste plus qu'une.» Inutile, pensons-nous, de continuer sur ce point. La prétendue «mode actuelle» se révèle un mode permanent de l'expression en Bande dessinée. L'innocence enfantine (du type des premiers Tintin, Superman ou Astroboy) est déconnectée de l'état historique de la Bande dessinée en général. Elle correspond à un choix de situation, plus ou moins délibéré, opéré par tel ou tel. 


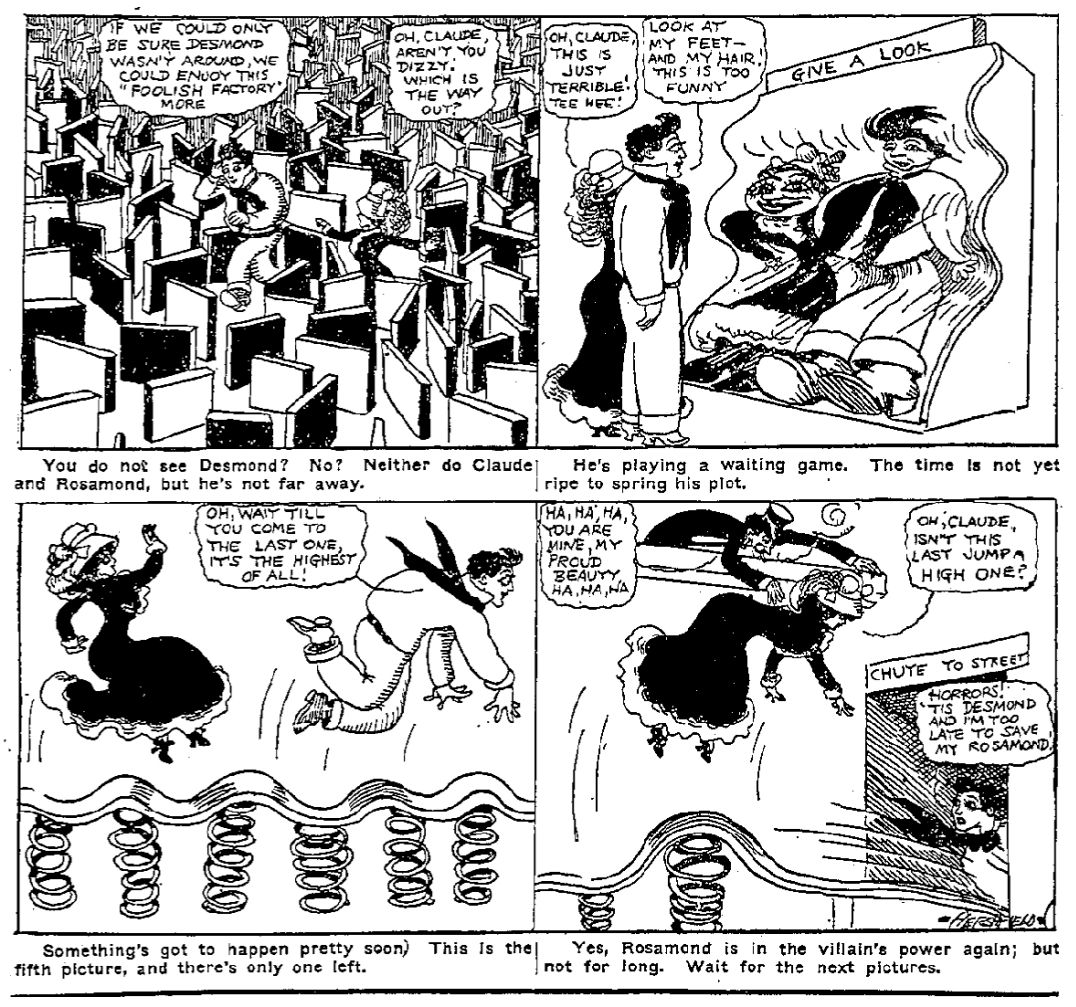

Harry Hershfield (c) New York Evening Journal Publishing Compagny, 1910.

L'autoprésentation, même aujourd'hui, dépasse de toute façon les caractères dominants que nous avons repérés. Au-delà de l'allure d'ensemble qui permet d'énoncer que la BD tient bel et bien un discours interprétatif dans sa propre réalisation, se trouve la singularité de l'œuvre. Nous voulons poser ce terme. Non pas une Bande dessinée reflet, symptôme, témoignage, illustration, etc.; non, une œuvre. Le double rapport d'autoprésentation - de soi comme unicité, voire singularité; de la BD en tant que telle ne suffit pas à faire œuvre, mais il y acquiert un sens crucial. Alors, la définition de la BD n'existe pas, mais elle est refaite et défaite chaque fois qu'une œuvre surgit.

\section{L'introduction de l'hétérogène}

51 Il devient loisible de privilégier des configurations, noter des récurrences, favoriser des parentés - quant à faire une synthèse, là n'est pas l'enjeu. On " pousse » sans doute une signification relativement à d'autres, mais il n'est en fait aucune définition qui ne soit exclusive. L'autoprésentation implique bien l'émergence de l'autre, le lecteur compris. Le préfixe ne doit pas conduire à un cercle fermé; la tautologie est branchée sur son contraire; sans quoi, rien. Nous revendiquons une certaine position, un goût si l'on veut; car nous sommes pour l'œuvre de Bande dessinée, plutôt que pour l'entertainment. À compter de là, foin du dogmatisme, où l'on comparerait un livre à une conception préalable du neuvième art. Comme dans les devinettes pour enfants, le jeu des sept erreurs, vous vous rappelez? Ce que nous recherchons n'est pas l'idéal-type, mais la Bande dessinée qui change (de) la Bande dessinée. Pour comprendre ce qui se passe audelà de la présentation, il vaut peut-être mieux la connaître. La chose n'est pas certaine à tout coup, mais s'il s'agit de contribuer à relancer la critique, cernons des significations. 
Voici, donc, un index sans exhaustivité aucune, qui laisse toutefois assez à méditer. Quelques moments où s'inventent une parole et un trait différents.

L'altération commande en quelque sorte une introduction - au sens, aussi, du mot en anglais - de l'hétérogène ${ }^{69}$. Un autre qui délimite l'opus, et, parfois, l'ouvre à d'autres vents. Ainsi, la BD se définit par la bande (le jeu était inévitable), en faisant passer d'autres figurations parmi elle. Il est frappant que la dernière aventure de Tintin, inachevée, règle d'étranges comptes avec les plasticiens. Dans Tintin et l'Alph-Art, le capitaine Haddock, toujours en quête de reconnaissance sociale, finit par se convertir à l'art contemporain. Il achète une sculpture alphabétique, un $H$, malgré les conseils contraires de la Castafiore, qui n'a toujours pas retenu le vrai patronyme de son ami le «marin pêcheur » et lui conseillait donc d'autres lettres. Cet enthousiasme nouveau met Tintin sur la piste de faussaires, qui placent sur le marché des œuvres indûment attribuées aux maîtres du présent et du passé. L'intrigue s'interrompt juste avant que les criminels n'essaient de « transformer [Tintin] en César ${ }^{70}$ ».

Dans L'T̂le noire et au début de L'Or noir, Tintin a déjà eu affaire à des faux-monnayeurs. Cette fois, le faussaire travaille dans « une véritable usine à faux tableaux... et imités à la perfection $^{71}$ ». « Décidément, nous nageons dans l'art !... », n'hésite pas à dire Tintin ${ }^{72}$. La plongée de l'action et des personnages dans une histoire de liquidités artistiques situe constamment par ironie l'ambition de la BD. Hergé, lui-même collectionneur de Roy Lichtenstein, aura plus tard inspiré l'une des dernières toiles du pop-artiste, Tintin Reading. Il place ici sa production dans un espace semi-mondain, où la récupération de la "culture populaire» côtoie la falsification des originaux. Tintin devait-il se « transformer » en faux art ? Vraisemblablement pas. Le héros s'en serait sorti. Ni faux, ni vrai art au sens des Monet ou Gauguin «imités", la BD d'Hergé a renoncé à la «perfection » de la peinture - authentique ou non. La sous-catégorie de l'alph-art ne sert dans l'histoire qu'à masquer un trafic. Mais elle pourrait correspondre à la signature d'Hergé, dont le pseudonyme vient justement des initiales inversées GR (pour Georges Rémy). L'abondance de codes cachés et messages cryptés dans Tintin nous incite à lire une affirmation derrière les crayonnés et les croquis : pour RG, l'alph-art de la BD est juste autre que les deux formes de l'art, le vrai falsifiable et le faux parfait.

Avec d'autres réponses, un problème identique se condense chez Enki Bilal. La Trilogie Nikopol $^{73}$ reprend les figurations picturales et sculpturales des dieux égyptiens, dont l'hétérogénéité interne homme/ animal est doublée par le pouvoir de possession qu'exerce Horus sur Nikopol. Le tout est rendu dans un style graphique qui choisit de décaler la Bande dessinée vers les beaux-arts. Le résultat fait alors signe vers une pensée du composite, de l'impureté, de l'incompatibilité malgré tout ${ }^{74}$, dont la Bande dessinée est la logique expression.

Dans ces parages, Frank Miller choisit manifestement une assomption des comics, au détriment de la grande tradition artistique. Dans Elektra Lives Again, l'aveugle Matt Murdock apparaît chez lui devantun réseau de couleurs transformé en Mondrian ${ }^{75}$. La toile n'est qu'une référence sous-jacente, elle est incluse dans la BD mais n'est pas une substance supérieure. C'est plutôt un accident, une interruption. Chez Miller, la Bande dessinée se recroise non par un défaut de culture, mais parce qu'en est assurée d'emblée la possibilité infinie. Le film noir, la gravure, les comics, le théâtre, le roman, la série télévisuelle sont aussitôt aspirés dans le vertigo de l'œuvre. Dans le même Elecktra, un policier dubitatif regarde sortir du cimetière un personnage dont l'étonnante allure contraste avec toute l'esthétique graphique de l'album. On dirait un collage, et il s'agit en 
effet de l'introduction furtive de Carmen Cru, la vieille mégère qu'a inventée Lelong. Déplacement de figure, d'esthétique et de registre - Elektra Lives Again est un long thrène, bien loin a priori de l'humour grinçant et dévastateur de la chère Carmen. Par là, Miller casse l'unité stylistique. Il démontre la facticité nécessaire à la constitution de l'œuvre. Pareille introduction reprend et libère la convention des mangas, qui, à la suite du père fondateur Osamu Tezuka, rendent les émotions violentes par une brutale distorsion des traits faciaux des personnages. Une jolie fille pique un fard ou est vexée, et son visage devient temporairement une caricature. L'exigence d'une présence externe dans l'élaboration opératoire est sans doute figée par son encodage. Mais les conventions ne procèdent pas de l'éternité; elles naissent et meurent, et nombre de mangakas contemporains n'ont que faire de la déformation rituelle. Miller en reprend le fonctionnement, en restitue le danger pour les doctrines de la cohérence. Un geste comparable lui fait inventer That Yellow Bastard ${ }^{76}$. Le corps d'un jeune meurtrier nanti est détruit par lepolicier intègre qui l'arrête. Après d'innombrables interventions chirurgicales, il ressuscite sous une nouvelle forme. Exactement défiguré, le psychopathe est rond et difforme dans une Bande dessinée où presque tout est anguleux, carré, pointu. Il est encore singularisé par sa couleur jaune au sein d'un livre autrement en noir et blanc. L'autre, ici, est chargé d'un supplément de présence par l'effectivité de son surnom. That Yellow Bastard est une insulte qui pourrait venir d'une pièce de Shakespeareautant que du monde noir de Sin City. Mais yellow est aussi jaune, littéralement. Que la première Bande dessinée américaine soit intitulée The Yellow Kid ne manque pas de reveniràl'esprit. Cet enfant jaune, hilareet sympathique trouve son contre-type dans l'homme jaune, répugnant et criminel. Alors qu'il inscrit son entreprise au cœur de l'art américain, Frank Miller fait ressortir le danger et la part d'ombre qui obscurcissaient déjà l'âge de l'innocence.

Un souci comparable de récriture de l'histoire se trouve dans The Sentry, de Jae Lee et Paul Jenkins, la mini-série qui fut peut-être la plusspectaculaire parmi les comics mainstream des dix dernières années ${ }^{77}$. Le passé occulté de Robby Reynolds refait surface en lui sous forme de souvenirs épars. Ces fragments mémoriels sont rendus par des couvertures et des planches de comics d'un autre temps. Des années 1960 à 1980, Jae Lee fait voir ce passé d'après les différents graphismes qui ont dominé les publications Marvel (styles Kirby, Romita Sr, Miller). L'écoulement temporel est en porte à faux avec la description physique des personnages, qui gardent une certaine jeunesse. Ce désaccord introduit au cœur de l'objet les insurmontables contradictions entre la continuité d'aventures commencées il y a plus de quarante ans et leur historicité cumulative. L'œuvre, en ce point, s'étend, par-delà la singularité de Sentry, jusqu'à « l'univers Marvel ». L'œuvre des comics superhéroïques renouvelés est l'introduction dans le réel d'une dimension fantastique supplémentaire, qui n'a pas fonction de diversion (comme Superman à l'origine) mais de démultiplication de l'expérience sociale. Nouant le pessimisme de Franck Miller au ton sacrificiel qui imprégnait le travail de Stan Lee, Jack Kirby et Steve Ditko, les deux auteurs font de Sentry un personnage qui engendre l'annihilateur Void, et qui choisit de disparaître une seconde fois dans l'oubli. L'oblative oblitération, certes positive, témoigne en creux de l'occulte pourtant maintenu dans les comics. Renonceraitelle au divertissement, la BD n'entraîne-t-elle pas toujours un retrait, un certain refus du monde, même au cœur du prétendu réalisme? La vieille critique contre l'activité antisociale de la Bande dessinée recouvrerait une actualité inattendue. 


\section{Adaptations et spécificités}

57 On l'aura compris, en tout cas, la fin de l'autoprésentation n'est pas elle-même. Le refus de lire ce que la BD peut dire et montrer a pour contrepartie erronée de ne chercher que la requalification de l'art par lui-même. Une réflexion sur la critique de l'œuvre à travers elle (et notre lecture!) peut mener ailleurs. Plusieurs des textes courts amorcés après cette longue présentation tentent ce risque; d'autres non. Le but était plutôt, dans ce retour sur la critique, de poser, de proposer une considération pour la BD qui puisse radicalement modifier le discours qu'elle suscite. Au lieu de demander à la Bande dessinée de jouer les indics (sur l'économique, le politique, le sémiotique...), au lieu de lui reprocher tout ce qu'elle ne sait pas faire (une symphonie, un tableau, une tragédie...), regardons ce qu'elle peut. Maintenant que son édiction est mieux affirmée, il est l'heure des comparaisons. Rapprochons la BD de ses adaptations filmiques, et rien n'assure que le cinéma soit plus puissant. L'un d'entre nous avait consacré un article à la vogue de transpositions, par Hollywood, des grands superhéros ${ }^{78}$. La liste des échecs s'est depuis lors allongée, avec Hulk ou Fantastic Four, autant de longs métrages où l'aventure graphique est comptée pour rien, où l'intrigue sentimentale est encore réduite. Pour le redire dans le langage qui vient d'être tenu, l'auto-présentation n'est pas vue, pas lue et rien ne vient prendre sa place dans le passage au grand écran. Bref, on garde le spectaculaire, à défaut de tout ce qui lui conférait de la signification. Pour être juste, il faudrait ajouter que la sérialité crée de facto une matière qui manquait au départ. Ainsi, l'approfondissement est envisageable, surtout si l'équipe change peu. Spiderman 2 et le deuxième X-Men réussissent làoùleurs « prequels »restaient vagues. La simple condensation d'une histoire à l'autre donne un sentiment comparable à l'émotion de lecture. Mais il faudrait plus d'affranchissement encore. Comme dans la recombinaison complète des faibles historiettes de Superboy que donne la série télévisée Smallville. Face à une Bande dessinée légère, la déformation très profonde et la grande sérialité rendent hommage aux puissances des comics. Dans un genre différent, Lucas et Spielberg ont su capter une capacité de la Bande dessinée - quitte, parfois, à faire s'absenter le cinéma, ou, du moins, l'entraîner vers les confins de l'imagerie et la fantasmagorie.

Le cinéma, à peu près aussi ancien que la Bande dessinée, et, autant qu'elle, violemment attaqué, a désormais acquis un statut particulier, une place dans la production reconnue des formes et des idées. Le qualificatif de «neuvième art » est au fond risible, car trop optimiste, trop loin d'une légitimation qui reste fort partielle; ou alors quasi nationaliste (le mangaka star au Japon, la ligne claire en Belgique). Les échecs à répétition de la transposition cinématographique directe peuvent entretenir l'idée que le point de départ n'en valait pas la peine. Ils pourraient encore, de même que les succès locaux entrânent une relecture des sources originales, aider à penser la spécificité de la Bande dessinée.

Celle-ci apparaît lorsque le sens de l'adaptation se modifie, lorsque la BD s'approprie l'un de ses autres. Le cas est patent avec la littérature. Comme au cinéma, la conduite de la narration réaliste a été décisive pour le récit de la Bande dessinée, en France et Belgique essentiellement - de loin en loin, dans les nouvelles générations américaines et les mangakas marginaux. Mais, d'encore plus près, il arrive à la $\mathrm{BD}$ de prendre le pas de la littérature, puis la quitter dans une fidélité à l'ambition de l'œuvre plus qu'à ses rugosités factuelles. 

trois instants magnifiques. David Mazzucchelli fait de La Cité de verre de Paul Auster unénigmatique graphic novel, plusélusifque le roman ${ }^{79}$. De longs passages du texte d'Auster sont consacrés au langage et aux théories successives sur le rapport entre le mot et le fait, la langue et le réel. Tout cela se trouve dit, par des personnages, des livres, le narrateur, de telle sorte qu'un continuum métalinguistique se constitue. Par définition, la narration de BD, bien qu'elle implique en général le langage verbal, ne peut bien sûr y demeurer entière. Mazzucchelli opte pour des pages presque toujours quadrillées, où une parole est dite dans la case par un objet incongru. Dans le monologue de l'ancien « enfant sauvage », une légère déviation du phylactère fait sortir les mots d'une autre bouche que celle qui devrait les prononcer. Le jeune homme est un pantin, porteur d'un discours intrus, et le flux de phrases devient indépendant, passant d'un vortex à un chapeau ou un oiseau. Le langage est séparé de l'énonciateur, simple masque, comme celui du tragédien grec figuré en Charon. Le passage du langage, intransitif, est une activation de la part blanchotienne de Paul Auster. Le discours se transforme sous nos yeux et dans l'inquiétante régularité du quadrillage graphique, métamorphose du gratte-ciel ou de la grille de prison.

61 donna du Woyzeck de Büchner ${ }^{80}$. Dans une figuration presque abstraite, la dernière scène du drame rend les sillons ouverts dans l'étang par Woyzeck, qui veut à la fois se laver du sang de son crime et faire disparaître son couteau. Tandis que les pages précédentes étaient dominées par des taches noires, des zones grisées ou des bulles emplies de texte, le fond disparaît progressivement, le contour des bulles s'efface. Ne restent plus que les silhouettes multipliées de Woyzeck et ses traces dans l'eau, qui semblent communiquer à travers l'espace infranchissable des cases. La page suivante, la dernière, donne en résolution un entrelacs de lignes formant un visage à travers les six cases en puzzle. Le surgissement de la Sainte face, venue du poème d'André Frénaud, renvoie à l'ouverture, où un visage morcelé était visible selon plusieurs gros plans. Marbeuf opère, par le moyen de la Bande dessinée, un rapprochement critique entre littérature et littérature, Büchner et Frénaud. Il y parvient sans inféodation, en inscrivant les lignes du texte, de l'eau, du crayon dans une nouvelle disposition. La fin ouverte de la pièce fragmentaire de Büchner ouvre sur le blanc de la page, le début du drame, le poème, l'œuvre.

Le dernier exemple, lui aussi impressionnant et limite, sera tiré du Château d'olivier Deprez, d'après Kafka ${ }^{81}$. Exercice hors norme, cetalbum est le résultat de dizaines de gravures sur bois, où la matérialité de l'écorce participe à l'image. À la toute fin, il n'y a presque rien, $\mathrm{K}$ peut-être sortant de la scène. Les rayures dans le bois demeurent, comme ces lignes dynamiques qui escortent les personnages quand on leur veut donner l'impression de vitesse. C'est le bois même, celui de la cabane largement montrée, celui des bûches qui sont plus tôt réunies pour le feu. On n'arrive pas au château, jamais vraiment; pourtant, il reste, cela, le concret du matériau, ou une trace, dont le sens n'est pas sûr. Étrange BD assurément, qui fait sauter les conventions et délivre une lecture critique de soi, de Kafka, de l'entêtante matérialité de la vie.

63 Nous parlons après un siècle et plus de bandes dessinées, et ce point où nous croyons être doit bien avoir quelque retentissement sur nos envies. Assurément, la BD peut être ramenée à ce qui la précède; puisque presque tout le monde l'a fait... Et si les comics ne se contentaient pas d'exprimer le secret, s'ils fabriquaient aussi d'autres mystères, apportaient d'autres interprétations ? Et si l'énigme, bien souvent, venait d'une simplicité à quoi l'on ne devrait pas s'arrêter? Nous ne voulons pas décrire froidement. Oui, nous

Labyrinthe, 25 | 2006 (3) 
souhaitons le dire : la Bande dessinée est à même de faire œuvre, de se réformer à partir de là. La grandeur de la BD, ne l'exagérons pas; ne la minimisons pas non plus. Tant pis pour les partisans du tout confort et du délassement; tant pis pour les amateurs qui voudraient trouver dans la bédé la neutralisation artistique. Eh oui, tout ne se vaut pas. Là où la bande se construit plus qu'elle ne se déroule gentiment, nous trouvons la force d'une critique de l'ouvre. Ce nouveau registre est-il indispensable? Pas forcément. Disons quand même qu'il nous paraît préférable à beaucoup de babils, ritualisés, schématiques.

Peut-être aussi que la Bande dessinée a maintenant besoin d'un discours tiers, qui suive la trace de son autoprésentation et rompe l'ordre de l'art dans un passage au seul écrit. Un discours tiers, qui la questionne autrement, qui l'irrite, qu'elle puisse mépriser ou impliquer dans sa pensée. En littérature, où le tiers espace n'est pas assuré du tout, où il est fait au contraire du substrat langagier et conceptuel - en littérature, la croissance du corps étranger critique a influé sur le textualisme, et sur la tentative d'échappatoire dans le biographique. Action et réaction. Malgré la concordance temporelle, la phase actuelle de la Bande dessinée, portée à l'introspection multiforme, pourrait, elle, naître du tiers critique défaillant. Les créateurs ont toujours dit et montré ce qu'ils pouvaient, mais arrive un temps où il suffit que cela s'oublie régulièrement. Et l'on retourne plus franchement sur soi, sur son histoire. Par enthousiasme, l'œuvre court le risque d'être un peu trop envahie par la diction de sa nécessité. Textualisme et biographisme se rejoindraient. Il nous paraît certain qu'en tout état de cause, la Bande dessinée empruntera plus tard d'autres voies sans rapports, malgré les oiseaux de mauvais augures ou les nostalgiques affectés. Depuis notre époque pourtant, il serait dommage que se perde encore une fois le savoir de la Bande dessinée. De l'effort, gardons acte par un renouvellement critique. Soyons toujours renégats, rétifs aux habitudes ancrées de la célébration univoque, la détestation générale, l'interprétation distanciée. Un peu critiques, un peu voyous; pourquoi pas?

Voici les adresses électroniques des auteurs: 1d79[at]cornell.edu et renaud.pasquier [at]gmail.com

\section{NOTES}

1. Ce livre compile les arguments que Wertham avait développés dans les années précédentes lors de conférences à travers les États-Unis. L'inquiétude à l'encontre des comics et des bandes dessinées est assez symptomatique de l'après-guerre en Occident et a abouti à des codes de censure ou des « lois de protection de la jeunesse », comme celle promulguée en France en 1949. Sur la controverse française au sujet de la $\mathrm{BD}$, on peut se reporter à l'article très complet de Richard I. Jobs, «Tarzan under Attack: Youth, Comics and Cultural Reconstruction in Postwar France ", French Historical Studies, $n^{\circ}$ 26-4, 2003, p. 687-725.

2. Voir les arguments d'Harry Morgan, Principes des littératures dessinées, Paris, Éditions de l'An 2, 2003, dans sa deuxième partie, consacrée aux "attaques contre les littératures dessinées ", et surtout sa lecture du bouquin de Wertham sur son site, à l'adresse : http://www.sdv.fr/pages/ adamantine/wertham.htm.

3. Qui s'attachait à faire apparaître le roman de Flaubert comme un livre « édifiant ». 
4. «Le mot voyou a un rapport essentiel avec la voie, la voirie urbaine, la voirie de la cité ou de la polis, et donc avec la rue, le dévoiement du voyou consistantàfaire un mauvais usage de la rue,àcorrompre la rue, à traîner dans les rues [...]. Le voyou est à la fois inoccupé, parfois au chômage, et activement occupé à occuper la rue, soit à "courir les rues", à n'y rien faire, à traîner, soit à faire ce qu'on ne doit pas faire normalement, selon les normes, la loi et la police, dans les rues et sur toutes les autres voies. " Jacques Derrida, Voyous, Paris, Galilée, 2003, p. 197-198.

5. Jean-Marie Apostolidès propose à l'endroit de Tintin le qualificatif de «surenfant ». Tintin et le mythe du Surenfant,éditions Moulinsart, 2003.

6. Voir Harry Morgan, op. cit., p. 156-164 : «Bande dessinée et enfance, histoire d'un préjugé ».

7. Tous trois publiés à L'Association, le premier en 2000, dans la collection "Ciboulette " (un second tome est à paraître), le deuxième dans la même collection, en 4 volumes, de 2000 à 2003, et le dernier,

dans la collection « Esperluette », en six volumes, de 1996 à 2003.

8. C'est l'explication avancée par Harry Morgan, op. cit., p. 157.

9. Ce concept de fétichisme et ses migrations (de l'ethnologie à la psychanalyse en passant par le marxisme) est examiné par Giorgio Agamben dans Stanze. Plus précisément, voir Giorgio Agamben, «Dans le monde d'Odradek. Euvre d'art et marchandise ", trad. Y. Hersant, Rivages Poche, Paris, 1998 (1977), p. 63-104.

10. Les forums des sites Internet, ainsi que les blogs, représentent aujourd'hui un autre espace où s'épanouit, pour le meilleur et pour le pire, la parole du fan.

11. Eisner/Miller, conversazione sul fumetto (traduction italienne), Bologna, Kappa Edizioni, 2005.

12. "Questions de méthode", dans Critix - Analyse et critique de la bande dessinée, $\mathrm{n}^{\circ} 8$ ("Les discours technique, socio-économique et encyclopédique», p. 9-22), n 9 («L'archivisme», p. 5-27), $\mathrm{n}^{\circ} 10$ (« Le discours critique », p. 11-34), 1999.

13. Paris, PLG, 2005 (pour la dernière révision). L'ouvrage est illustré par Lewis Trondheim.

14. Paris, 1975 , p. 37-60.

15. Harry Morgan reprend rapidement les points essentiels du chapitre en question et s'attache à les réfuter - de manière parfois trop rapide - dans ses Principes des littératures dessinées, livre II, p.185-189. La critique menée par l'école de Francfort aurait beaucoup marquée Wertham. Elle informe assurément le long texte de Gershon Legman publié en 1948 sous forme d'article («The Psychopathology of the Comics ", Neurotica, n 3, 1948, p. 3-30; traduit partiellement dès 1949 dans Les Temps modernes, "Psychopathologie des"comics" ", $n^{\circ} 43$, p. 916-933), et repris dans Love \& Death. A Study in Censorship, New York, Breaking Point, 1949.

16. C'est-à-dire surtout le jazz. Voir T. W. Adorno, Le Caractère fétiche de la musique, trad. fr. de Christophe David, Paris, Allia, 2001 (éd. originale 1938).

17. La question de ce type de discours critique est abordée par André Simon, dans son article « Approches idéologiques », dans Europe n 720, «La Bande dessinée », avril 1989, p. 88-95.

18. Alain Moreau, collection « Textualité », 1976 (éd. originale 1971).

19. Voir André Simon, «Le monde musulman dans la BD », dans $I^{\text {er }}$ Symposium international sobre la historieta, Barcelone, 1985, repris dans« L'Arabo-musulman dans la BD », Cahiers rationalistes, $\mathrm{n}^{\circ}$ 431, avril 1988.

20. On renvoie notamment à l'analyse d'Harry Morgan sur le livre de Dorfman et Mattelart, à l'adresse http://www.sdv.fr/pages/adamantine/dorfman.htm.

21. «À condition qu'elle porte la griffe d'un grand styliste, une paire de bottes vaut Shakespeare. Et tout à l'avenant : une Bande dessinée qui combine une intrigue palpitante avec de belles images vaut un roman de Nabokov [...] un slogan publicitaire efficace vaut un poème d'Apollinaire ou de Ponge. [...]. Ce n'est plus la grande culture qui est désacralisée, [...] - ce sont le sport, la mode, le loisir qui forcent les portes de la grande culture. [...] Par une sorte de confusion joyeuse, "on" élève la 
totalité des pratiques culturelles au rang des grandes créations de l'humanité », Alain Finkielkraut, La Défaite de la pensée, Gallimard, 1987, p. 138-139. (C'est nous qui soulignons.)

22. Certes, tout comme Adorno, qu'il admire, Steiner n'évoque pas la Bande dessinée en tant que telle, mais on le sait éternel défenseur de la "haute culture " aujourd'hui menacée, et contempteur de la médiocrité culturelle contemporaine, signe et facteur de la nouvelle barbarie. On ne donnera pas de référence précise, l'ensemble des travaux de Steiner étant traversé par cette profonde inquiétude.

23. Explicitée par Adorno dans son texte "Culture Industry Reconsidered», dans The Culture Industry: Selected Essays on Mass Culture, Londres, Routledge, 1991.

24. Sous le masque des voyous-sauvageons-barbares.

25. Paris, L'Association, collection « Éprouvette », 2005.

26. Que Boltanski publie ce texte sur la Bande dessinée dans le premier numéro (donc fortement symbolique) des Actes de la recherche en sciences sociales est un fait en soi révélateur. On y lit la volonté de marquer l'étendue du domaine ouvert à l'analyse sociologique, et en particulier celui des biens culturels (Bourdieu publie des textes sur la mode et sur Flaubert).

27. Concédons que le cas d'Eco est particulier. D'abord, parce qu'il s'est penché - certes brièvement - sur les formes, les tâches et les limites des discours tenus sur la Bande dessinée, dans une réflexion non sans proximité avec celle que nous présentons ici, qui appelle à une attention aux œuvres elles-mêmes ("Quattro modi di parlare di fumetti », que l'on pourra lire en ligne sur le site de la revue, extrait du $n^{\circ} 9$ à l'adresse http://www.fucine.com/network/ fucinemute/core/index.php?url=redir.php?articleid=153; ledit article est issu d'un ouvrage consacré à Claire Brétecher, Claire Bretécher: il disegno del fumetto, a cura de La Cappella Underground / Comune di Trieste / Associazione italo-francese). Ensuite, parce qu'on ne peut lui dénier le statut de pionnier, plusieurs articles signés de son nom ayant incontestablement fait date : sur Les Peanuts de Schultz, sur Superman, ou sur Steve Canyon (tous rassemblés, en italien, dans le recueil Apocalittici e integrati, Milano, Bompiani, 1964. Et on accordera à Eco qu'il s'attache à envisager la Bande dessinée de la même façon que les autres arts. C'est cette "façon ", justement, qui nous paraît problématique : celle du touche-à-tout érudit et malin, capable de faire flèche de tout bois, et qui finalement exalte bien plus sa propre ingéniosité que l'objet qu'il étudie.

28. Paris, Minuit, 1978.

29. Paris, Seuil, 1957.

30. «[...] on ne peut fonder une véritable science de l'œuvre d'art qu'à condition de s'arracher à l'illusio ", Bourdieu, Les Règles de l'art - Genèse et structure du champ littéraire, Paris, Seuil, coll. «Points Essais », 1998, p. 376-377.

31. Le premier est le Celeg (Centre d'études des littératures d'expression graphique) et le second la Socerlid (Société civile d'études et de recherches des littératures dessinées).

32. Pierre Couperie, Proto Destefanis, Édouard François, Maurice Horn, Claude Moliterni, Gérald Gassiot-Talabot, Bande dessinée et figuration narrative, Musée des arts décoratifs, 1967.

33. Il y a, nous semble-t-il, des consensus sur les « grandes œuvres ", les mutations essentielles, les mouvements déterminants dans chacun des domaines disciplinaires touchant les autres arts, un terrain commun sur lequel débats et querelles s'enflamment, qui peut fort bien être remis en question, mais qui a du moins une consistance absente dans la Bande dessinée.

34. Thierry Groensteen l'a bien montré dans son article «Définition et origine: des rapports entre la sémiologie de la Bande dessinée et son étude historique ", disponible sur son site

Internet à l'adresse : http://www.editionsdelan2.com/groensteen/sequence1/ definition\&origine.html

35. Benoît Peeters, Lire la Bande dessinée, Paris, Champs Flammarion, 2002 (éd. originale Casterman, 1998), p. 10-11. 
36. Will Eisner, Comics \& Sequential Art, Tamarac, Poorhouse Press, 1985 ; Graphic Storytelling \& Visual Narrative, Tamarac, Poorhouse Press, 1996.

37. L'Art invisible [Understanding Comics], trad. fr. de Dominique Petitfaux, Paris, Vertige Graphic,1999 [Kitchen Press, 1991].

38. Il faudrait aussi, comme le suggère Benoît Peeters, intégrer l'Essai de physiognomonie de Rodolphe Töpffer, et en première place, chronologiquement, à cette liste d'ouvrages théoriques rédigés par des auteurs.

39. Paris, Hachette, 1972.

40. Lyon, Presses universitaires de Lyon, 1985. À ne pas confondre avec l'ouvrage de Benoît Peeters qui porte le même titre.

41. Paris, Puf, coll. « Formes sémiotiques », 1999.

42. «Pour commencer, cette étude devrait renouer avec la perspective mimétique condamnée par le structuralisme, l'analyse d'une bande dessinée se ramenant à la question: par quels moyens passe la restitution du monde de la fiction? Ce faisant, on ne succomberait pas à l'illusion référentielle; on chercherait au contraire comment opère cette illusion. On couvrirait par là-même la totalité des aspects formels des littératures dessinées et des mécanismes producteurs de sens. »

43. Voir les dénégations de Peeters : «Malgré les apparences, le présent ouvrage n'entretient que des rapports assez lointains avec la sémiologie de la Bande dessinée qui s'illustra dans les années soixante-dix ", Lire la Bande dessinée, op. cit., p. 12 ; c'est nous qui soulignons.

44. Jan Baetens mène ainsi des recherches qui tentent d'articuler sémiotique et «études culturelles", Les Principes des littératures dessinées d'Harry Morgan comportent de larges développements d'ordre historique, et l'on est surpris de voir l'historien Jean-Paul Gabilliet regarder Groensteen comme le représentant d'une théorie « essentialiste » (Jean-Paul Gabilliet, Des Comics et des Hommes. Histoire culturelle des comic books aux États-Unis. Nantes,éd. du Temps, 2005, p. 14), quand ledit Groensteenplaide pour une étude plus complète de la Bande dessinée s'inspirant des acquis de l'histoire culturelle (« Définition et origine ", art. cit).

45. On songe notamment (il y aurait d'autres exemples) aux acquis du Système de la Bande dessinée (op. cit.) de Thierry Groensteen, qui permettent d'appréhender avec plus de finesse et de précision les moyens de la bande dessinée. Notre propos n'est pas ici d'évaluer ou de discuter ses propositions, ou d'autres, on se contentera donc de renvoyer à la lecture de l'ouvrage.

46. «Un nouveau départ pour l'étude des comics", Acta Fabula, printemps 2005 (vol. 6, n 1), URL : http://www.fabula.org/revue/document880.php.

47. Source d'équivoques possibles : ainsi lorsque Benoît Peeters affirme que le discours critique « domine de manière presque absolue le champ des études sur la Bande dessinée » (Lire la Bande dessinée, op. cit., p. 11), il faut comprendre l'ensemble des travaux monographiques qui relèveront bienplus de l'histoire, sous des formes variées (étude biographique, thématique, etc.).

48. Pour une évocation détaillée et argumentée des différentes tentatives pour élaborer une critique de la Bande dessinée, on se reportera justement au premier numéro de ladite revue, plus précisément à l'article de Jean-Christophe Menu, "Avant-garde et ultracritique ", dans L'Éprouvette ${ }^{\circ} 1$, op. cit., p. 173-186.

49. www.du9.org.

50. Voir le site http://www.english.ufl.edu/imagetext.

51. Nous entendons par là que l'idée d'œuvre engage un «effet-auteur »: si elle n'est aucunement réductible à une intention à elle antérieure, elle n'est pas non plus pensable indépendamment d'une figure, aussiévanescente fût-elle,àqui la rapporter.

52. «Textures. Premier épisode », Paris, L’Association, janvier 2006, p. 27-40.

53. Ibid., p. 29.

54. Publié chez Dargaud.

55. Trois séries publiées chez Delcourt. 
56. Publié chez Dupuis.

57. Publié à L'Association.

58. Publié chez Delcourt.

59. Sept tomes à l'heure qu'il est, publiés par L'Association, les six premiers dans la collection "Mimolette ». Ils sont réunis en 2005 en un volume de la collection «Ciboulette ». Le septième, sorti en 2005, se singularise par l'usage de l'aquarelle, et par la présence d'un titre, La Java bleue, qui l'isole des autres.

60. Tous publiés à L'Association, collection "Côtelette", ainsi que les deux autres, Parapluie (2003) et Caravan (2005).

61. Art. cit., p. 28. Où Sfar reformule l'assertion baudelairienne, selon qui « le meilleur compte rendu d'un tableau pourra être un sonnet ou une élégie » (dans «À quoi bon la critique ? ", dans Baudelaire, Critique d'art, Paris, Folio essais, 1992, p. 77-79). Baudelaire a lui-même un prédécesseur enFriedrich Schlegel: «La poésie ne peut être critiquée que par la poésie. Un jugement sur l'art qui n'est pas lui-même une œuvre d'art [...] n'a pas droit de cité au royaume de l'art ", dans Ph. Lacoue-Labarthe et J.-L. Nancy, L'Absolu littéraire, théorie de la littérature du romantisme allemand, Paris, Seuil, 1978, p. 95.

62. Plusieurs, oui, car les livres les plus audacieux rendent justement compte de la fragmentation. Voir cette page extraordinaire d'Approximativement où le Je-Lewis sort de soimême tous ses je, comme d'une poupée gigogne (Lewis Trondheim, Approximativement, Paris, Cornélius, 1995).

63. Voir Frank Miller et David Mazzucchelli, Batman. Year One, New York, DC Comics, 2005.

64. Stan Lee et Jack Kirby, Fantastic Four n 2, Marvel Comics, 1961, p. 18.

65. Stan Lee et Jack Kirby, Fantastic Four $n^{\circ}$ 4, Marvel Comics, 1962, p. 8.

66. Stan Lee et Jack Kirby, Fantastic Four $n^{\circ} 10$, Marvel Comics, 1962, p. 5-7. Fatalis est nommé «Dr Doom » dans la version originale.

67. George Herriman, « Krazy Kat » du 16 avril 1922.

68. Harry Hershfield, Desperate Desmond, était un strip publié par The New York Evening Journal. Quoique la série soit assez oubliée aujourd'hui (et en attente d'une réédition), elle connut un succès certain. Plusieurs films adaptèrent la saga au grand écran en 1911 et 1912. Trois ans après la fin des aventures de Desmond, un soixante-dix-huit tours transposait la Bande dessinée dans un univers sonore et musical. Outre l'illustration reproduite dans ce numéro, on pourra entendre l'étrange sketch de Fred Duprez sur le site http://www.wsu.edu/ campbelld/amlit/1910m.html.

69. Le choix de ce terme est lié à l'étude que Stéphane Delorme nous promet depuis longtemps à propos du cinéma. Mais la valeur de l'hétérogène ici n'engage bien sûr que nous.

70. Hergé, Tintin et l'Alph-Art, Bruxelles, Casterman, 1986, p. 42. Rappelons que cet album n'a pu être achevé par son auteur.

71. Id., p. 39.

72. Id., p. 11.

73. Enki Bilal, Bruxelles, 2005.

74. Certains hôtes « rejettent » la possession par un dieu; tous, même Nikopol, sont usés par une visitation trop prolongée.

75. En France : Frank Miller, Lynn Varley, Elektra le retour, Comics USA, 1991.

76. Publié en France sous le titre Cet enfant de salaud, Montreuil, Rackham, 1997.

77. En France : Paul Jenkins, Jae Lee et al., Sentry, 2 vol., Saint-Laurent du Var, Panini comics et Marvel France, 2001.

78. Laurent Dubreuil, «Spiderman se défile », Balthazar. Revue d'analyse du cinéma contemporain, $\mathrm{n}^{\circ}$ $6,2003$.

79. Le livre est publié en France sous les noms de Paul Auster, Paul Karasik et David Mazzucchelli, Cité de verre, Arles, Actes Sud, 1995.

80. «La Sainte face ", dans Le Cheval sans tête, $\mathrm{n}^{\circ} 1,1996$. 
81. Fremok, 2003. 